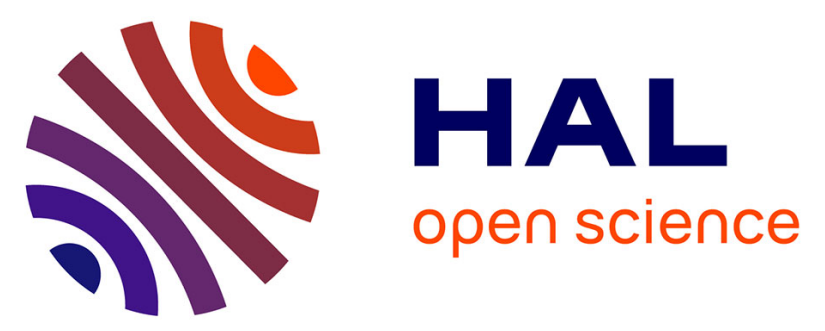

\title{
Heterochromatin renewal after release from growth arrest controls genome-wide transcription re-activation in S.cerevisiae
}

Pritha Bhattacharjee, Hrvoje Galic, Pauline Vasseur, Marta Radman-Livaja, Ana Hrgovčić

\section{To cite this version:}

Pritha Bhattacharjee, Hrvoje Galic, Pauline Vasseur, Marta Radman-Livaja, Ana Hrgovčić. Heterochromatin renewal after release from growth arrest controls genome-wide transcription re-activation in S.cerevisiae. 2021. hal-02390899v2

\section{HAL Id: hal-02390899 \\ https://hal.science/hal-02390899v2}

Preprint submitted on 29 Oct 2021

HAL is a multi-disciplinary open access archive for the deposit and dissemination of scientific research documents, whether they are published or not. The documents may come from teaching and research institutions in France or abroad, or from public or private research centers.
L'archive ouverte pluridisciplinaire $\mathbf{H A L}$, est destinée au dépôt et à la diffusion de documents scientifiques de niveau recherche, publiés ou non, émanant des établissements d'enseignement et de recherche français ou étrangers, des laboratoires publics ou privés. 


\title{
Heterochromatin renewal after release from growth arrest controls genome-wide transcription re-activation in S.cerevisiae
}

\author{
Pritha Bhattacharjee ${ }^{1,2 \#}$, Hrvoje Galici ${ }^{1,2 \#}$, Ana Hrgovčić ${ }^{1,2 \#}$, Pauline $\operatorname{Vasseur}^{1,2,3}$ and \\ Marta Radman-Livaja ${ }^{1,2^{*}}$
}

${ }^{1}$ Institut de Génétique Moléculaire de Montpellier, UMR 5535 CNRS, 1919 route de Mende, 34293 Montpellier cedex 5, France;

${ }^{2}$ Université de Montpellier, 163 rue Auguste Broussonnet, 34090 Montpellier, France.

${ }^{3}$ current address : CIRAD, Campus international de Baillarguet, UMR 117- ASTRE "Animal,

Santé, Territoires, Risques \& Écosystèmes", TA A-117/E, Bât. G bureau 212, 34398

Montpellier cedex 5, France

*Corresponding author

\# Equal contribution

\begin{abstract}
The budding yeast SIR complex (Silent Information Regulator) is the principal actor in heterochromatin formation, which causes epigenetically regulated gene silencing phenotypes. The dynamics of the SIR complex during the cell cycle are however not well understood. It is consequently still not known how the SIR complex is maintained and/or restored after genome replication and cell division, and how the underlying silenced state is transmitted form one cell generation to the next. We used the tag switch RITE system to measure genome wide turnover rates of the SIR subunit Sir3p during and after exit from growth arrest caused by nutrient depletion. Our results show that Sir3p subunits have high rates of exchange immediately after release from growth arrest. "Maternal" Sir3p is consequently almost completely replaced with newly synthesized Sir3p in subtelomeric regions by the end of the first cell cycle after release from growth arrest. The sudden increase in the off rate of Sir3 upon release from growth arrest leads to SIR complex instability that is exacerbated in strains with sub optimal amounts of newly synthesized Sir3p. Unexpectedly, heightened SIR complex instability in these Sir3p "hypo-morphs" has global effects on gene expression with faster reactivation of hundreds of euchromatic genes upon exit from growth arrest.
\end{abstract}




\section{Introduction}

Heterochromatin in budding yeast is a transcriptionally repressive structure located at the silent mating type loci $(H M R$ and $H M L)$, telomeres and rDNA repeats. The essential component of this structure is the non-histone protein complex SIR (Silent Information Regulator), which consists mainly of Sir2p, Sir3p and Sir4p. Sir4p scaffolds the complex while Sir2p functions as an NAD-dependent H4K16 deacetylase, providing a high-affinity binding site for Sir3p which recruits Sir4p (for review see (1)). In the classical polymerization model, SIR components are first recruited to silencer regions by a combination of silencerbinding factors (ORC -Origin Recognition Complex, Rap1p and Abf1p). The SIR complex then spreads from the nucleation site (silencer) through cycles of deacetylation and nucleosome binding, which continue until the SIR complex reaches boundary elements that prevent unwanted spreading to transcriptionally active regions (for review see (2) ).

It has been shown that over-expressed Sir3p can be incorporated into existing heterochromatin (3). However, beyond this bulk measurement, the locus-specific dynamics of the chromatin bound SIR complex within and from one cell generation to another have not yet been measured. How heterochromatic SIR complexes exchange their components during the cell cycle and how they are distributed to daughter chromatids after replication has important implications for how heterochromatic states are maintained and whether they may be inherited. The maternal SIR complex has to be disassembled during replication and if heterochromatin is to be restored on both daughter strands, the SIR complex has to be reformed on both strands to pre-replication levels.

Another open question is how chromatin bound complexes that epigenetically determine gene expression states, like the SIR complex, respond to environmental challenges such as nutrient depletion. Indeed, under unfavorable conditions, yeast cells stop growing and eventually enter the stationary phase, which lasts until depleted nutrients are restored. The stationary phase is characterized by transcriptional reprogramming, spatial reorganization of the genome and a 300 fold decrease in protein synthesis rates (4). While the organization of the SIR complex in stationary phase has been described, the dynamics of the SIR complex during and following exit from the stationary phase are still poorly understood (5). These questions have motivated us to investigate the dynamics of the SIR complex during and upon release from the stationary phase. We chose to focus on the Sir3p subunit because Sir3p is the limiting factor that determines the extent of SIR complex polymerization and the location of SIR complex boundaries (6-8).

Our strategy was to use the Recombination-Induced Tag Exchange (RITE) method (9, 10) to map genome wide Sir3p turnover rates with the goal to better understand the 
mechanisms of heterochromatin maintenance and find out whether the SIR complex is "inherited" on newly replicated daughter chromatids.

Our results show that subtelomeric Sir3p occupancy is reduced and Sir3p turnover is slow in arrested cells. Sir3p exchange rates increase dramatically immediately after release from growth arrest resulting in the almost complete replacement of "maternal" Sir3p with the newly synthesized Sir3p by the end of the first cell cycle after release. This suggests that at least the Sir3p subunit of the SIR complex is not inherited and therefore does not contribute to the epigenetic inheritance of the silenced state in subtelomeric regions and silent mating type loci in these conditions.

We propose that the sudden increase in Sir3p off rates upon release from growth arrest transiently destabilizes the SIR complex, which causes a temporary increase in gene expression at silent mating type loci and an increase in the number of cells that are unresponsive to $\alpha$ factor.

Finally, our RNA-seq datasets normalized to S.pombe mRNA, show that gene expression is restored faster on most low and moderately transcribed genes upon exit from growth arrest suggesting that heterochromatin integrity is important for the regulation of gene expression programs in sub-optimal growth conditions.

\section{Results}

\section{Sir3p exchange dynamics during and after release from growth arrest}

We used the RITE tag switch system developed by Fred van Leeuwen (10) to construct the Sir3p "tag switch" module. The genomic SIR3 locus was engineered with a Cterminal tag switch cassette containing loxP sites, and the 3xHA and 3xT7 tags separated by a transcription termination signal, and the hygromycin resistance gene (Figure 1A). The host strain carries the CreEBD78 recombinase, which is activated upon estradiol addition. After Cre-mediated recombination of LoxP sites, the HA tag on Sir3p is switched to the T7 tag. Recombination efficiency is assessed by growth on hygromycin plates, and is typically $~ 97 \%$ if arrested cells are treated with estradiol for at least $16 \mathrm{hrs}$.

Cells were incubated in glucose rich medium (YPD) for $48 \mathrm{hrs}$ until they stopped dividing, and they were then kept in stationary phase for $16 \mathrm{hrs}$ after estradiol addition to allow for the tag switch to complete in $\sim 97 \%$ of cells. It has been shown that after $48-64 \mathrm{hrs}$, at least half of the cells in the population have the characteristics of quiescent cells i.e. the ability to retain viability and reproductive capacity in the absence of nutrients. Consequently 
our experiments record the average behavior of a population of cells exiting stationary phase in which at least $50 \%$ of the cells are quiescent (11).

We use the term "stationary phase" as shorthand for 48-64 hrs. cultures of cells that have stopped growing due to nutrient depletion and caution the reader that cells in our cultures are not necessarily equivalent to stationary phase cells from 7 day old cultures described in the literature $(5,11)$. Arrested cells were incubated with estradiol for $16 \mathrm{hrs}$ to induce the Cre mediated tag switch. Anti-HA (old Sir3p) and anti-T7 (new Sir3p) chromatin immuno-precipitations were performed in parallel at regular intervals before and after Cre activation.

ChIP-seq datasets (normalized to average genome read density as described in Materials and Methods) of "old" and "new" Sir3p showed that Sir3p dynamics are similar at all heterochromatic loci, including silent mating type loci and subtelomeric regions (Figure 1 B-D and replicate experiment in Supplementary Figure S1). Since Sir3p with the "old" tag stops being produced after the tag switch, we can determine Sir3p OFF rates during and after exit from stationary phase by measuring how "old" Sir3p enrichment changes after the tag switch from the slope of the linear fit of the decrease in "old" Sir3p enrichment at time points during stationary phase and during the first cell cycle after release. Likewise, the Sir3p ON rate can be estimated from the initial rate of increase in "new" Sir3p enrichment up to the point when "new" Sir3p enrichment reaches equilibrium. The slope of the linear fit of the change in "new" Sir3p enrichment after release is approximately equal to the Sir3p ON rate even though it actually represents the ON/OFF rate ratio of "new" Sir3p, because the OFF rate of "new" Sir3p is initially negligible because most of the bound Sir3p is the one with the "old" tag in the early time points after the tag switch.

Our measurements show that the old Sir3p stays bound to chromatin in stationary phase cells even after the tag switch because of a slow OFF rate of $4 \%, 0.6 \%$ and $0 \%$ decrease in Sir3p-3xHA enrichment per hour and a slow ON rate of 3.5\%, 3\% and 3.5\% increase in Sir3p-3xT7 enrichment per hour for sub telomeres, HML and HMR, respectively (Figure $1 \mathrm{C}-\mathrm{E})$ (rates in $\Delta$ enrichment $\% / \mathrm{hr}(\Delta \mathrm{E})$ were calculated according to the equation: $\Delta E=\left(2^{|b|}-1\right) * 100$, were $\mathrm{b}$ is the slope of the linear fit from Figure 1 C-E). This results in very slow exchange rates of $-1 \% / \mathrm{hr},+2.1 \% / \mathrm{hr}$ and $+3.5 \% / \mathrm{hr}$ for subtelomeres, HML, and HMR respectively. Overall Sir3p enrichment after $64 \mathrm{hrs}$ in stationary phase relative to genome average is decreased $\sim 2$ to 4 fold compared to the prestationary midlog phase as shown in the replicate experiment in Supplementary Figure S1 (B-C). After release into fresh media, old Sir3p completely disappears by the first cell doubling and is replaced by new Sir3p. Old Sir3p is completely replaced by new Sir3p by the 
end of the first cell cycle after release because ON rates increase by $45 \%$ to $55 \%$, while OFF rates increase by $9 \%$ to $77 \%$ at subtelomeres and silent mating type loci, respectively, compared to stationary phase Sir3p exchange rates (Figure 1C-E). The enrichment in new Sir3p reaches steady state after the first division after release resulting in lower Sir3p occupancy relative to values before the stationary phase. New Sir3p enrichment after the $12^{\text {th }}$ doubling is still 3 fold lower than old Sir3p immediately after release and $\sim 7$ fold lower than Sir3p in mid-log cells (Figure 1B-C and Supplementary Figure S1B-C).

The observed pattern of Sir3p binding dynamics in cell populations after exit from stationary phase suggests that the existing Sir3p is rapidly removed from heterochromatin when cells resume growth before the first cell division and is mostly replaced with new Sir3p by the end of the first cycle after release. Sir3p replacement at silent mating type loci is faster than at subtelomeric loci, probably because of the stronger SIR complex nucleation capacity of HML and HMR silencers compared to subtelomeric silencers (Figure 1D-E, Supplementary Figure S1D-E).

The heat map in Figure 1B revealed an unexpected new Sir3p-T7 enrichment at tRNA genes. New, T7 tagged Sir3p appears to be significantly enriched at all but 20 tRNA genes immediately upon release from stationary phase. The biological significance of this binding is not clear as the replicate experiment from Supplementary Figure S1 using a different anti-T7 antibody (a monoclonal one versus the polyclonal one in Figure 1), while confirming Sir3p-T7 binding to tDNAs, shows enrichment levels that are 8 to 16 fold lower than in Figure 1.

Heterochromatin containing old Sir3p is destabilized during the stationary to growth phase transition when new Sir3p amounts are limited

The SIR complex appears to be unstable in the first cell cycle after exit from stationary phase, thus making heterochromatin potentially permissive to transcription during that period. Unless Sir3p is redistributed evenly genome-wide, the rapid decrease of old Sir3p density in heterochromatin during the first cell cycle after release is probably not due to Sir3p binding elsewhere in the genome because we did not detect new Sir3 peaks during this period. The old Sir3p is probably degraded because the drop in heterochromatin bound Sir3p coincides with a decline in total cellular old Sir3p, which has already been reduced to $14 \%$ of its midlog levels in stationary phase (Figure 2A).The drop in Sir3p levels in stationary phase probably occurs because SIR3 gene expression and Sir3p protein synthesis rates decrease more than (Figure 3F) Sir3p protein decay rates although all three processes are significantly slowed down in stationary cells. Note that even though SIR3 mRNA levels are low in stationary phase, there is still some Sir3p synthesis since we can detect a slow but positive 
"new" Sir3p-T7 binding rate to heterochromatic regions (Figures 1 and S1 C-E). The apparent half-life of Sir3p in stationary phase from the time point "before the tag switch" (after estradiol addition) to the "after switch" time point in the western blot in Figure 2A was estimated to be $\sim 28$ hrs (Figure 2B), which fully accounts for the $90 \%$ decrease is Sir3p levels during $48 \mathrm{hrs}$ in stationary phase. The disappearance of old Sir3p proteins below levels detectable by western blot by the time of the first cell doubling is accompanied by a slow and gradual increase in new Sir3p which is detectable around $90 \mathrm{~min}$ after release (i.e. 2 hrs. before the first doubling) (Figure 2A). SIR3 gene expression of the Sir3p-3xT7 construct (resulting from tag switch recombination) increases gradually after release from arrest but remains low even by the first division (Figure 3F). Low SIR3 gene expression probably accounts for the delay in new Sir3p appearance on the blot (Figure S2). Incidentally, the appearance of new Sir3p on western blots at $90 \mathrm{~min}$ after release coincides with the time when new Sir3p occupancy starts exceeding old Sir3p occupancy in subtelomeric regions (Figures 1 and S1). The half-life of old Sir3p after release from stationary phase is estimated at $\sim 110$ min (Figure 2B). Considering that most yeast proteins have a half-life between 60 and $150 \mathrm{~min}(12)$, old $\mathrm{Sir} 3 \mathrm{p}$ is probably degraded by the usual cellular protein degradation machinery, which resumes its activity at pre-stationary phase rates upon release.

\section{The SIR complex is transiently destabilized upon exit from stationary phase}

Since a sudden increase in Sir3p exchange rates immediately after exit from stationary phase could potentially destabilize the SIR complex and compromise heterochromatin function, we tested the silencing potential of SIR complexes after exit from stationary phase using the " $\alpha$-factor test", described below (Figure 3).

MATa (Mating Type a) cells will respond to the presence of $\alpha$-factor (mating pheromone $\alpha$ ) by arresting in G1 and forming a "shmoo" if the silent mating type locus $H M L$ (containing the genes for mating type $\alpha$ ) is stably heterochromatinized with the SIR complex. If $H M L$ is not fully silenced, cells will behave like pseudo-diploids and will not respond to $\alpha$ factor. Log-phase cells should therefore predominantly respond to $\alpha$-factor and become shmoo. On the other hand, we expect that populations exposed to $\alpha$-factor immediately upon exit from stationary phase will have a higher fraction of cells that form buds. This is exactly what we observe: 52\% of cells with a Sir3-3XHA tag (same strain as the one used in Figures $1,2, \mathrm{~S} 1$ and S2, except that the tag switch was not induced in this experiment) do not form shmoon upon release from stationary phase, which is nearly twice the rate of budding cells in midlog populations (29\%) exposed to $\alpha$-factor. The high proportion of cells that were unresponsive to $\alpha$-factor in midlog conditions was however surprising. Suspecting an adverse effect of the 3xHA epitope tag on the stability of the mRNA or the stability or function of the 
protein, we performed the same experiment with the parent strain with an untagged Sir3 (WT1) or the Sir3 RITE tag switch strain isolated after the tag switch (Sir3-3XT7) (Figure 3A-C). Insensitivity to $\alpha$ factor does increase 20 fold after exit from stationary phase in cells with the untagged Sir3 compared to exponentially growing untagged cells but the overall effect is greatly diminished compared to tagged Sir3p cells since only $3.25 \%$ and $0.15 \%$ of the cell population exiting stationary phase and midlog cells, respectively, did not shmoo in the presence of $\alpha$-factor (Figure 3C). The degree of insensitivity to $\alpha$ factor depends on the size and type of the epitope tag and the linker sequence that connects the tag to the $\mathrm{C}$ terminus of Sir3p (Figure 3A). While a triple HA tag substantially reduces Sir3p functionality, the triple $\mathrm{T} 7$ tag almost completely obliterates it with $77 \%$ and $97 \%$ of cells insensitive to $\alpha$ factor before and after stationary phase, respectively. The triple tag may either destabilize the mRNA or the protein or interfere with the ability of Sir3p to interact with other SIR complex subunits or with its ability to bind to chromatin. Increased mRNA instability resulting in lower amounts of tagged Sir3p in the cell is however probably not the cause of the increased insensitivity to $\alpha$ factor in tagged midlog cells since RNA-seq datasets normalized to an external S.pombe RNA spike-in (Figure 6) show that midlog SIR3 mRNA levels are comparable in all three strains. The decrease in SIR3 mRNA levels during the first cell cycle after exit from stationary phase does however explain the relative increase in $\alpha$ factor insensitivity during this period compared to midlog cells in all three strains (Figure 3F). Sir3p enrichment at nucleation sites in subtelomeric and silent mating type loci is 6 to 8 fold lower in the 3xHA strain used in Figures 1 and S1 compared to an untagged WT strain (8). The extent of Sir3p spreading from nucleation sites is however similar between the untagged and tagged strain (Figure S2). This suggests that the triple tag impairs Sir3p binding to nucleation sites but does not interfere with SIR polymerization. The absence of a 6xHis tag in the linker region upstream of the 3xHA tag impairs Sir3p function even more, while Sir3p with a single HA or T7 tag are both functionally indistinguishable from untagged Sir3p (Figure 3D).

The increased inability to form shmoon upon stationary phase exit is not linked to a defect that is independent of Sir3p. Cells that can shmoo in log phase in the presence of $\alpha$ factor irrespective of the presence Sir3p due to a HML $\alpha M A T a$ double deletion, also shmoo with $100 \%$ efficiency upon release from stationary phase even in the absence of Sir3p (Figure 3E).

We also tested whether Sir3p turnover rates, measured in ChIP-seq experiments in Figures 1 and S1, were affected by 3xHA and 3x-T7 tags. We have seen above that single HA and T7 tags do not impair Sir3p function. However, RITE tag switch ChIP experiments with 
the single HA and T7 tags did not yield sufficient amounts of DNA to prepare NGS libraries of good quality. We consequently had to compare Sir3p exchange rates at silent mating type loci in the Sir3-3xHA to $3 x$ T7 RITE strain with Sir3p exchange rates in the Sir3 1xT7 to 1xHA RITE strain (Figure S3) using ChIP-qPCR. Our Sir3 ChIP qPCR experiments show that except for the on rate at HMR-E, exchange rates are comparable between the two strains suggesting that neither the triple HA nor the T7 tag alter the dynamics of Sir3p subunit exchange within the SIR complex (Figure S3C). We consequently conclude that the Sir3p exchange rates of all heterochromatic loci measured by ChIP-seq with the Sir3-3xHA to 3xT7 RITE strain in Figures 1 and S1 are close to wt Sir3p exchange rates.

We used the same SIR3 construct with a 3xHA tag under the control of the galactose inducible GAL1 promoter as in (8) to test how SIR3 overexpression affects Sir3p ON and OFF rates after exit from stationary phase (Figure 4A). SIR3 overexpression after exit from stationary phase extends the subtelomeric Sir $3 p$ binding domains by as much as $15 \mathrm{kbp}$, as previously observed for SIR3 overexpression in midlog cells(8) (Figure 4B). Cells were first allowed to grow exponentially in galactose to induce Sir3p synthesis and allow SIR complex formation. Cells were then left to enter the stationary phase after galactose depletion. Since galactose depletion in stationary phase stops Sir3p production in this system and dextrose represses the GAL1 promoter, we can estimate genome-wide Sir3p ON and OFF rates after exit from stationary phase in conditions of SIR3 overexpression simply by performing two parallel ChIP-seq time-courses in cells released from arrest into galactose or dextrose media, respectively. The OFF rate is directly estimated from the rate of decrease of Sir3p enrichment after release from stationary phase into dextrose. The ON rate can then be calculated from the increase in Sir3p enrichment after release in galactose and the OFF rate measured in dextrose according to the formula $O N(\% / h r)=\left(2^{\left(b_{i}+b_{d}\right)}-1\right) * 100$, where $b_{i}$ and $b_{d}$ are the slopes of the linear fits for the rates of increase in Sir3p enrichment in galactose and decrease in Sir3p enrichment in dextrose, respectively.

Our calculations show that Sir3p ON rates at subtelomeres and HMR are respectively 50\% and 2 fold higher when SIR3 is overexpressed (Figure 4 F and J). Sir3p enrichment has reached equilibrium only $1 \mathrm{hr}$ after release into galactose, suggesting that SIR complex renewal does not directly depend on DNA replication or cell division but is likely driven by the rate of Sir3p synthesis. Sir3p OFF rates on the other hand are comparable in cells with wt Sir3p levels and in Sir3 overexpression cells. It is however important to note that the process of old Sir3p removal occurs independently of Sir3p synthesis as evidenced by the complete disappearance of chromatin bound old Sir3p by the second doubling upon release into dextrose or raffinose in which Sir3p is not produced anymore (Figure 4H, I). In other words, 
as in stationary phase cells (Figure 2A), the removal of old Sir3p from heterochromatin in growing cells is not "driven" by its replacement with new Sir3p.

Continuous Sir3p presence after exit from stationary phase is necessary for the maintenance of low level transcription of subtelomeric genes

The $\alpha$ factor test from Figure 3 suggests that the sudden increase in Sir3p exchange rates after release from the stationary phase transiently destabilizes the SIR complex, which temporarily de-represses HML and HMR and turns a fraction of the cells in the population into pseudo-diploids. As we have seen above, the size of the cell fraction rendered insensitive to $\alpha$ factor is inversely proportional to the level of Sir3p enrichment at silent mating type loci, which are significantly reduced in strains with the triple HA or T7 tag.

We therefore decided to take advantage of the Sir3p "hypo-morph" phenotype of the strains with the triple HA or T7 tag to assess how varying levels of Sir3p enrichment in subtelomeric and silent mating type loci affect gene expression in these regions, after release from stationary phase by performing RT-qPCR experiments with the following strains: the SIR3 overexpression strain (oeSir3), two WT strains with untagged Sir3p (WT1 and WT2), the Sir3-3xHA RITE strain before the tag switch, the Sir3-3xT7 RITE strain after the tag switch, and finally a $\operatorname{Sir} 3 \Delta$ strain (Figure 5).

We chose six genes in proximity (800bp to $1300 \mathrm{bp}$ ) to subtelomeric SIR complex nucleation sites (XCS): AAD15, ARR3 (2400bp from XCS), PAU19, PAU22, PAU4 and PAU6. We also used HMRa2 from the HMR locus a s a positive control. PAU genes have very similar sequences so the qPCR primers used in the experiment detect more than one target as indicated in Figure 5. Likewise, the HMRa2 gene is identical to the MATa2 gene (our strains are MATa). Moreover, HMRa2 is actually a 119 aa C-terminal truncation of HMLa2. Consequently, our qPCR primers will detect cDNA from all three loci. The nonsubtelomeric gene SSL2 that is not regulated by the SIR complex, was used for internal normalization of $\mathrm{Ct}$ values (Figure 5B).

Surprisingly, the SIR complex does not seem to have much of an effect on the expression of the tested subtelomeric genes as their expression profiles do not show significant differences between WT and SIR3 $\Delta$ or Sir3 hypo-morph strains. Additionally, the bulk of the HMRa2/MATa2/HMLa2 signal in all the strains except oeSir3, where the MAT locus is deleted, probably comes from the expression of MATa2 in the MAT locus. Consequently, the de-repression of HMRa2 and HMLa2 in Sir $3 \Delta$ cells is masked by the signal form MATa2. 
The expression profiles of subtelomeric genes and HMRa2 are markedly different in the SIR3 over expression strain, however. Sir3p synthesis in this strain is controlled by galactose. Sir3p is therefore not even produced at the low levels found in WT cells because galactose is depleted from the media in stationary phase. Surprisingly, all tested subtelomeric genes were downregulated 4 to 16 fold relative to SSL2 immediately after release from stationary phase when new Sir3p synthesis has not yet begun, but when old Sir3p has already started coming off chromatin. Gene expression returns to midlog levels 90 min after exit from stationary phase by which time, according to our results from Figure 4, newly synthesized Sir3p has accumulated to levels sufficient for the reconstitution of the SIR complex. HMRa2 is repressed throughout the time course, probably because Sir3p enrichment at the HMR locus in midlog was very high and old Sir3p had not been depleted after exit from stationary phase before new Sir3p started binding to HMR.

Our results from Sir3 $\Delta$ cells suggest that the SIR complex is not responsible for the "default" low expression of subtelomeric genes. However, our results from oeSir3 cells show that once the SIR complex binds in the proximity of these genes, its continuous presence at the locus is needed to maintain this low "default" level of transcriptional activity, which drops substantially if Sir3p is depleted.

The idea that continuous Sir3p binding is necessary to maintain low levels of transcription at subtelomeric genes is supported by the Sir3p ChIP qPCR experiment shown in Figure 5C. The drop in Sir3p binding during stationary phase and in the early time points after release coincides with the decrease in mRNA expression. mRNA returns to midlog levels when Sir3p binding is restored 90min after exit from stationary phase. In the Sir3$3 x H A$ strain, on the other hand, Sir3p binding appears to increase during stationary phase and immediately after release. Sir3p eventually goes back down to midlog levels by the end of the first cycle after release. The apparent relative increase in Sir3p enrichment is puzzling. Given that it coincides with the increase in Sir3p exchange rates, we propose that higher Sir3p turnover makes the HA tag more accessible to antibodies resulting in higher ChIP yields and an apparently higher Sir3p occupancy.

\section{Transcription at hundreds of genes is restored faster after exit from stationary phase when Sir3p levels are too low}

Entry and exit from stationary phase elicit dramatic global changes in the cellular transcription program $(13,14)$. Our results on the effect of Sir3p turnover on SIR complex function (Figure 3) also suggest that heterochromatin is transiently destabilized and heterochromatic genes are temporarily de-repressed immediately after exit from stationary 
phase. Since de-repression of silent mating type loci creates a pseudo-diploid phenotype with a diploid specific transcription program(15), we decided to investigate how Sir3p dynamics during the stationary to growth phase transition might affect genome wide gene expression levels.

In order to be able to directly compare mRNA levels between time points and different yeast strains, we performed RNA-seq experiments with external "spike in" normalization using RNA from S.pombe as described in Materials and Methods (Figure 6). We measured mRNA dynamics during exit from stationary phase in the following strains: two WT strains with untagged Sir3p (WT1 and WT2), the Sir3-3xHA RITE strain before the tag switch, the Sir3-3xT7 RITE strain after the tag switch, and $\operatorname{Sir} 2 \Delta$ and $\operatorname{Sir} 3 \Delta$ strains.

We identified genes whose gene expression trajectory during the time course is most dependent on the presence of Sir3. First, we divided genes into four clusters according to their average midlog gene expression levels in the WT1 strain. Incidentally, Figure 6A shows that the average midlog gene expression levels of all tested strains in each cluster are similar to WT1. Second, we determined the Pearson correlation between the gene expression time course of WT1 and every other tested strain for each gene. Finally, in order to find the genes whose gene expression profiles in SIR3 $\Delta$ cells were the least similar to WT, we calculated the difference between the Sir3 $\Delta / \mathrm{WT} 1$ and WT2/WT1 correlations for each gene $(\Delta$ correlation $(\operatorname{Sir} 3 \Delta-\mathrm{WT} 2))$.We then ordered genes in each cluster by their $\Delta$ correlation(Sir3 $\Delta$-WT2). Genes in the quartile with the most negative average $\Delta$ correlation(Sir3 $\Delta$-WT2) in each cluster are the ones whose gene expression across the time course is the least similar between Sir $3 \Delta$ cells and both WT1 and WT2 cells. Conversely, the quartile from each cluster with an average $\Delta$ correlation(Sir3 $\Delta$-WT2) that is closest to 0 contains genes whose expression in $\operatorname{Sir} 3 \Delta$ cells is the most similar to either WT strain (Figure 6A).

We thus found 1449 genes whose gene expression dynamics after exit from stationary phase are the most affected by the absence of Sir3p or Sir2p, or by reduced amounts of Sir3p. The expression profiles of these genes averaged per cluster and per strain show that the main difference between WT and the other strains lies in the speed at which mRNAs go back up to midlog levels after a drop observed in stationary phase (Figure 6B). It takes on average one whole cell cycle for mRNAs to go back up to midlog levels in both WT strains. This happens much sooner in Sir $3 \Delta$ cells. Typically, mRNA levels are back up only 5 min after release from stationary phase. This happens somewhat later in the Sir3p "hypo morphs" -Sir3-3xHA, Sir33xT7- and Sir2 $\triangle$ cells: at the 30min time point, which is still $3 \mathrm{hrs}$ before mRNAs level off in WT cells. mRNAs are leveling off faster in SIR complex mutants and Sir3p hypo morphs 
even in the quartiles with genes whose expression is the most similar between $\operatorname{Sir} 3 \Delta$ and WT cells. The restoration of midlog mRNA levels in these non WT strains, is fastest for genes with the lowest expression. It consequently appears that the wt amounts of the SIR complex are needed to globally slow down the reactivation of transcription and delay the resumption of full transcriptional activity till the end of the first cell cycle after release from stationary phase. GO annotation analysis shows that genes that are the most affected by suboptimal SIR complex levels are involved in tRNA amino-acylation, protein transport and localization, nucleotide metabolism, and Golgi apparatus function. The least affected genes are mostly involved in ribosome function and biogenesis (Table S1).

We also looked at subtelomeric genes whose expression is more likely to be directly affected by changing Sir3p levels. Consistent with our RT-qPCR results from Figure 5 and previous reports(16), the wt levels of the SIR complex do not control the expression of most subtelomeric genes because we found only 16 genes out of 132 in our RNA-seq datasets that were located within $15 \mathrm{kbp}$ of subtelomeric SIR nucleation sites, whose gene expression profiles in SIR mutants and Sir3p hypo-morphs were significantly different from WT cells ( $\Delta$ correlation(Sir3 $\Delta$-WT2) $\leq-0.34$, Figure S4). As we mentioned above, external "spike-in" normalization of RNA-seq reads allows us to directly compare mRNA levels between different time points and different strains. We now see that, except for AAD15, midlog expression of these genes is not significantly different between WT and SIR mutant or Sir3p hypo-morphs as observed before(16). All 16 genes are however more expressed in the SIR3 "mutant" strains than in either WT strain when cells are in stationary phase and immediately after exit from stationary phase. The increase in expression observed in SIR3 mutants is only temporary and mRNA levels fall back to low midlog levels by the $1^{\text {st }}$ division after release. It consequently seems that the SIR complex, even when it is bound at wt levels, is not necessary for the silencing of subtelomeric genes in optimal growth conditions. It is needed instead to dampen transcription during the stationary phase and prevent a premature burst of activity early after release. It appears that the SIR complex keeps transcriptional activity low until the cell is ready for the complete re-establishment of its transcription program.

SIR dependent attenuation of transcription of at least some subtelomeric genes in stationary phase and after exit from stationary phase might have been expected because of their proximity to SIR bound loci. On the other hand, the fact that $\sim 1500$ euchromatic genes located far away from "canonical" SIR loci exhibit a similar SIR dependent delay in transcription re-activation, is more puzzling.

One possible explanation for the faster reactivation of transcription in the absence of the SIR complex is that transcriptional activity had not been fully shut down in stationary 
phase and might therefore be able to restart faster upon exit from arrest. If there was still lingering genome-wide transcription in stationary phase, we reasoned that the apparent mRNA half-lives in SIR mutants and hypo-morphs would be longer than in WT cells. For simplicity sake, we assumed that the decay pattern for most mRNAs is exponential, even though it is most likely more complex for a great number of mRNAs (17). Of course our prediction would only hold if the "true" mRNA decay rates are the same in all tested strains, which we hypothesized should be the case because they are for the most part determined by mRNA sequence and secondary structure, which are the same in all strains(18). We consequently determined apparent mRNA half-lives during stationary phase using the datasets from Figure 6.

We detect a genome-wide drop in mRNA abundance in stationary phase, as would be expected if global transcription activity has largely stopped during that period. We measured apparent mRNA decay rates and half-lives directly from the decrease in mRNA levels from the midlog to the stationary phase, as shown in Figure S5. Somewhat surprisingly and as opposed to decay rates in midlog cells (18), stationary phase mRNA decay rates are directly correlated with midlog mRNA levels meaning that the more an mRNA was abundant before stationary phase the shorter its half-life during stationary phase (Figure S5). However, with the exception of Sir $2 \Delta$ in which mRNA half-lives are globally $~ 50 \%$ longer, we did not observe any significant difference in mRNA half-lives between $\operatorname{SIR} 3 \Delta$ and $\operatorname{Sir} 3 p$ hypo-morph strains and WT strains.

\section{Discussion}

Our measurements of Sir3p dynamics within heterochromatin after exit from stationary phase show a dramatic increase in Sir3p exchange rates immediately after release from arrest (Figure 1). We show that Sir3p subunits exchange very slowly during stationary phase and heterochromatin is gradually depleted of Sir3p due to protein degradation, which is also slowed down relative to exponentially growing cells as we show in Figure 2. The supply of fresh nutrients triggers the reactivation of all cellular processes including faster Sir3p protein degradation and faster Sir3p turnover within the SIR complex. Consequently, all Sir3p proteins bound to subtelomeric and silent mating type loci are eventually replaced with newly synthesized Sir3p by the end of the first cell cycle after release. Budding yeast heterochromatin is therefore a highly dynamic structure that is continuously renewed throughout the cell cycle. Sir3p is not epigenetically inherited and does not facilitate the reconstitution of the SIR complex after replication, at least not in the first cell cycle after release from stationary phase. The RITE tag switch system cannot be used to differentiate between replication dependent and replication independent Sir3p dynamics in exponentially 
growing cells because Cre mediated recombination of epitope tags takes 16 hrs i.e. the length of 8 to 11 cell doublings. A different strategy that relies on fast and temporally controlled labelling of Sir3p should therefore be used to asses Sir3p dynamics in exponentially growing cells.

Even though the RITE strain with a Sir3-3xHA to Sir3-3xT7 tag switch that we used to measure genome-wide Sir3p exchange rates turned out to be a Sir3p hypo-morph with lower Sir3p enrichment at heterochromatic loci than in WT cells, we show in Figure S3 that the genome-wide Sir3p exchange rates measured in this strain are not significantly different from Sir3p exchange rates measured in a strain that does not exhibit the "hypo-morph" phenotype. Sir3p turnover rates are therefore not dependent on the quantity of Sir3p bound to chromatin. We consequently conclude that the Sir3p dynamics we observe in the Sir3-3xHA to $3 \times$ T7 RITE strain are a true reflection of Sir3p dynamics in WT cells in these growth conditions.

The $\alpha$ factor test for the gene silencing function of the SIR complex revealed that the sudden increase in Sir3p turnover upon exit from stationary phase that follows Sir3p depletion in stationary phase compromises SIR function in a small but significant fraction of the cell population. Rapid Sir3p turnover compounded with the depletion of Sir3p that happened during stationary phase appears to make the SIR complex relatively more permissive to transcription of silent mating type loci. If the supply of new Sir3p is not optimal as in the Sir3p hypo-morphs, SIR dependent silencing of silent mating type loci is 15 to 30 fold less efficient after exit from stationary phase and 200 to 500 fold less efficient in midlog cells compared to WT cells in the same conditions (Figure 3).

We found that the SIR complex directly controls the expression of only a small subset of subtelomeric genes and that its silencing activity is only apparent in stationary phase and shortly after exit from stationary phase (Figure S4). The molecular mechanism behind this strengthening of silencing activity is not clear, but it appears to be sensitive to Sir3 dosage and fluctuations in Sir3 levels. If Sir3p is depleted quickly as in the oeSir3 system, transcription actually drops abruptly but is also restored rapidly to its pre-stationary phase levels as soon as the SIR complex is restored (Figure 5). If Sir3p levels don't fluctuate as much and are sustained by continuous Sir3p turnover as is the case in WT cells, transcription activity seems to drop and recover more gradually over the course of the entire cell cycle (Figure S4).

The biological function of the SIR complex in subtelomeric regions has long been somewhat of a mystery. Results from this study and others argue against a primary role in the 
silencing of subtelomeric genes through position effect variegation (16) in exponentially growing cells where the absence of Sir3 has little effect on the expression of subtelomeric genes (Figures 5 and S4). It has also long been speculated that the SIR complex prevents deleterious inter-chromosomal homologous recombination between repetitive subtelomeric regions similar to its role at the rDNA locus (19). DuBois et al. (20) have however shown that telomeric recombination events are not more frequent in Sir3 and Sir2 mutants thus disproving that hypothesis. On the contrary the SIR complex stimulates the homologous recombination pathway during double strand break repair by promoting telomere clustering and bringing them close to each other(21).

In light of our results we speculate that the role of the SIR complex is not simply to exclude the transcription machinery from heterochromatic regions. Its continuous presence would instead be needed to "keep" the transcription machinery in subtelomeric regions and modulate its activity in response to growth conditions. If Sir3p is depleted from these regions, the transcription machinery would also be lost and Sir3p binding would have to be restored for the transcription machinery to be recruited again. This idea is supported by a study that used in vitro reconstituted heterochromatin substrates to dissect the mechanism of SIR mediated transcription silencing (22). They determined that transcriptional co-activators bind to promoters even in the presence of SIR and that the SIR complex inhibits the interactions between co-activators and their partners. In line with our hypothesis above, they also show that RNAPolII can bind to heterochromatin albeit inefficiently and that SIR prevents transcription elongation by the bound RNAPolII.

Surprisingly, our analysis of gene expression dynamics using RNA-seq with S.pombe RNA "spike in" normalization also revealed wide-spread fast reactivation of transcription of euchromatic genes, immediately after exit from stationary phase in SIR mutants and "hypomorphs". It is not clear why the SIR complex would have such a global effect on transcription reactivation since the vast majority of affected genes are not known to be directly or indirectly controlled by the SIR complex. One possibility is that transient loops between subtelomeric regions and distal targets could bring the SIR complex over target genes to act as a enhancer of silencing or transcription attenuator in certain growth conditions. Subtelomeric regions would then conceptually be similar to enhancers in higher eukaryotes except that instead of activating transcription they would repress it. Unlike enhancers though, their silencing activity would also have to be non-specific as they appear to indiscriminately target a great number of genes. These loops may be difficult to detect if they are short lived or if they only form in certain growth conditions such as under stress or during exit from stationary phase. A few of these bridges have recently been identified by Hi-C in midlog cells 
when Sir3p was overexpressed (23) and it will be very interesting to see if more can be detected in stationary phase cells. It is also not clear why the cell would need to have a mechanism that controls the speed at which transcription is reactivated although one could imagine that a controlled and gradual restart of cellular processes is preferable to an uncoordinated "every gene for itself" approach. Controlled cellular "awakening" probably ensures that all the components and all the nutrients necessary for the optimal functioning of the cell will be available in sufficient quantity and at the right time for the cell to optimally resume its growth.

Taken together, our results suggest that yeast heterochromatin may have a wider role in the control of gene expression programs than previously thought and future studies should further explore the molecular mechanisms behind this process.

\section{Materials and Methods}

Detailed Experimental Procedures are listed in the SI Appendix.

\section{Yeast Strains}

Genotypes and strain construction are described in the SI Appendix.

\section{Cell culture}

Cells were kept at $30^{\circ} \mathrm{C}$ in rich media for $48 \mathrm{hrs}$ until carbon source depletion and growth arrest. For the tag switch experiments estradiol was added and cells were incubated for another $16 \mathrm{hrs}$ before release. For the experiments without tag switch cells were released after $48 \mathrm{hrs}$ into fresh media and fixed with formaldehyde at indicated time points.

\section{Microscopy and image analysis}

Cells were injected under a $0.8 \%$ agarose/growth media layer in 8 -well glass bottom microscopy plates (BioValley) and visualized on a wide field inverted microscope for epifluorescence and TIRF acquisition (Nikon) under the HiLo setting.

\section{Chromatin Sonication and ChIP}

Cell walls were mechanically disrupted the entire cell lysate was sonicated. ChIPs were done as described in the SI Appendix using anti-HA (Abcam, ab9110 (lot\# GR32457073) and polyclonal anti-T7 (Bethyl A190-117A (lot\# A190-117A-7) (Figure 1) or monoclonal anti-T7 (Cell Signaling Technology, DSE1X (lot\#1)) (Supplementary Figure S1). Purified DNA was treated with RNAse A and used for NGS library construction.

\section{RNA isolation, RNA-seq libary construction and Reverse Transcription}

Cells were flash frozen in liquid N2 and total RNA was isolated from frozen cell pellets with Trizol and treated with DNAseI. RNA samples were then used for RT-qPCR or 
NGS library preparation with the Illumina TruSeq Stranded mRNA kit according to the manufacturer's protocol. Libraries were sequenced on the Illumina NextSeq550 (2x75bp) (Plateforme Transcriptome, IRMB, Montpellier, France).

$2 \mu \mathrm{g}$ DNAse-I treated and purified total RNA was reverse transcribed using 200 units of Superscript II (Invitrogen) with of $0.5 \mu \mathrm{g}$ N6 random primers.

\section{qPCR}

Primer sequences are listed in Table S2. Reactions were done with Platinum Taq polymerase and SYBR green mix(24) and data were collected on LightCycler 480 (Roche).

Cts were normalized as described in Figure Legeneds.

\section{NGS Input and ChIP library construction and Illumina sequencing}

DNA fragments were blunt ended and phosphorylated with the Epicentre End-itRepair kit. Adenosine nucleotide overhangs were added using Epicentre exo- Klenow. Illumina Genome sequencing adaptors with in line barcodes were then ligated using the Epicentre Fast-Link ligation kit. Ligated fragments were amplified using the Phusion enzyme Library pools were sequenced on the HiSeq 2000 (2x75bp) (Illumina) at the CNAG, Barcelona, Spain or the NextSeq550 (2x75bp) (Plateforme Transcriptome, IRMB, Montpellier, France).

\section{ChIP-seq and RNA-seq data analysis}

All analysis was done using in house Perl and R scripts available upon request.

Sequences were aligned to S. Cerevisiae genome using BLAT (Kent Informatics, http://hgdownload.soe.ucsc.edu/admin/). Read count distribution was determined in $1 \mathrm{bp}$ windows and then normalized to 1 by dividing each base pair count with the genome-wide average base-pair count. For Sir3p binding to genes from Figure 4, Input normalized read densities for each gene were aligned by the transcription start site. The median read density for each gene (from the tss to the end of the coding sequence) was determined for each transcript and the $\log 2$ of the median was determined for non-zero values. Medians with a value 0 were kept as 0 and signify that no sequences were detected for that gene in that particular dataset (time point).

RNA-seq normalized read densities for each gene were aligned by the transcription start site and divided into sense and antisense transcripts. The median read density was determined for each transcript as above. Intron regions were excluded from the calculation.

\section{Data availability}

NGS dataset were submitted to the NCBI GEO database. GEO accession numbers for ChIP-seq and RNA-seq datasets are

\section{Author Contributions}


$\mathrm{PB}$ and $\mathrm{AH}$ performed experiments in Figures 3, 5 and 6 and $\mathrm{HG}$ performed experiments in Figures 1, 2, 4, S1 and S3. PV performed the microscopy in Fig 3E. MRL, PB, $\mathrm{AH}$ and $\mathrm{HG}$ designed the experiments, MRL analyzed the data and wrote the manuscript with input from $\mathrm{PB}$ and $\mathrm{AH}$.

\section{Acknowledgments}

We thank Fred van Leeuwen and Kevin Struhl for yeast strains and plasmids. Thank you to Marta Gut and Julie Blanc from CNAG (Barcelona, Spain) and Véronique Pantesco (IRMB, Montpellier, France) for deep-sequencing services. We thank Virginie Georget (MRI, Biocampus, Montpellier) for her assistance with the microscope set-up. A previous version of this article is part of the doctoral thesis "Heterochromatin dynamics upon release from stationary phase in budding yeast" by Hrvoje Galic. This work was supported by the ERCConsolidator grant (NChIP 647618) (MRL).

\section{References}

1. M. Grunstein, S. M. Gasser, Epigenetics in Saccharomyces cerevisiae. Cold Spring Harb Perspect Biol 5 (2013).

2. M. R. Gartenberg, J. S. Smith, The Nuts and Bolts of Transcriptionally Silent Chromatin in Saccharomyces cerevisiae. Genetics 203, 15631599 (2016).

3. T. H. Cheng, M. R. Gartenberg, Yeast heterochromatin is a dynamic structure that requires silencers continuously. Genes Dev 14, 452-463 (2000).

4. J. N. McKnight, J. W. Boerma, L. L. Breeden, T. Tsukiyama, Global Promoter Targeting of a Conserved Lysine Deacetylase for Transcriptional Shutoff during Quiescence Entry. Mol Cell 59, 732-743 (2015).

5. M. Guidi et al., Spatial reorganization of telomeres in long-lived quiescent cells. Genome Biol 16, 206 (2015).

6. A. Hecht, S. Strahl-Bolsinger, M. Grunstein, Spreading of transcriptional repressor SIR3 from telomeric heterochromatin. Nature 383, 92-96 (1996).

7. $\quad H$. Renauld et al., Silent domains are assembled continuously from the telomere and are defined by promoter distance and strength, and by SIR3 dosage. Genes Dev 7, 1133-1145 (1993).

8. M. Radman-Livaja et al., Dynamics of Sir3 spreading in budding yeast: secondary recruitment sites and euchromatic localization. Embo J 30, 1012-1026 (2011).

9. M. Terweij et al., Recombination-induced tag exchange (RITE) cassette series to monitor protein dynamics in Saccharomyces cerevisiae. G3 (Bethesda) 3, 1261-1272 (2013).

10. K. F. Verzijlbergen et al., Recombination-induced tag exchange to track old and new proteins. Proc Natl Acad Sci U S A 107, 64-68 (2010). 
11. C. Allen et al., Isolation of quiescent and nonquiescent cells from yeast stationary-phase cultures. J Cell Biol 174, 89-100 (2006).

12. M. Auboiron et al., TrIPP- a method for Tracking the Inheritance Patterns of Proteins in living cells- reveals retention of Tup1p, Fpr4p and Rpd3L in the mother cell. iScience, 102075 (2021).

13. A. P. Gasch et al., Genomic expression programs in the response of yeast cells to environmental changes. Mol Biol Cell 11, 4241-4257 (2000).

14. M. J. Martinez et al., Genomic analysis of stationary-phase and exit in Saccharomyces cerevisiae: gene expression and identification of novel essential genes. Mol Biol Cell 15, 5295-5305 (2004).

15. J. E. Haber, Mating-type genes and MAT switching in Saccharomyces cerevisiae. Genetics 191, 33-64 (2012).

16. A. Ellahi, D. M. Thurtle, J. Rine, The Chromatin and Transcriptional Landscape of Native Saccharomyces cerevisiae Telomeres and Subtelomeric Domains. Genetics 200, 505-521 (2015).

17. C. Deneke, R. Lipowsky, A. Valleriani, Complex degradation processes lead to non-exponential decay patterns and age-dependent decay rates of messenger RNA. PLoS One 8, e55442 (2013).

18. C. Miller et al., Dynamic transcriptome analysis measures rates of mRNA synthesis and decay in yeast. Mol Syst Biol 7, 458 (2011).

19. S. Gottlieb, R. E. Esposito, A new role for a yeast transcriptional silencer gene, SIR2, in regulation of recombination in ribosomal DNA. Cell 56, 771-776 (1989).

20. M. L. DuBois, Z. W. Haimberger, M. W. Mclntosh, D. E. Gottschling, A quantitative assay for telomere protection in Saccharomyces cerevisiae. Genetics 161, 995-1013 (2002).

21. A. Batté et al., Recombination at subtelomeres is regulated by physical distance, double-strand break resection and chromatin status. EMBO J 36, 2609-2625 (2017).

22. A. Johnson, R. Wu, M. Peetz, S. P. Gygi, D. Moazed, Heterochromatic gene silencing by activator interference and a transcription elongation barrier. J Biol Chem 288, 28771-28782 (2013).

23. M. Ruault et al., Sir3 mediates long-range chromosome interactions in budding yeast. Genome Res (2021).

24. G. Lutfalla, G. Uze, Performing quantitative reverse-transcribed polymerase chain reaction experiments. Methods Enzymol 410, 386400 (2006). 


\section{Figure Legends}

Figure 1: A. Diagram of the Sir3p tag switch construct used in ChIP-seq experiments (top left). Bottom: Experiment outline. Cells were arrested by glucose depletion before the tag switch, induced with estradiol addition to stationary cells (recombination efficiency: 98.1\%). Cells were then released from arrest with addition of fresh media and allowed to grow for one to five doublings (monitored by OD measurements). Cell aliquots were fixed with $1 \%$ formaldehyde for $20 \mathrm{~min}$ at times indicated below the diagrams and anti-HA and anti-T7 (polyclonal) ChIPs were performed on sonicated chromatin. B. Heat map of new Sir3p (T7 tag) enrichment over old Sir3p (HA tag) during and after exit from stationary phase, at all yeast telomeres (30kbp from chromosomes ends). Time points are aligned by the ARS Consensus Sequence (TEL-ACS) located in telomeric silencer regions (SIR complex nucleation sites). White arrows show tRNA genes where new Sir3p binds after exit from stationary phase. Silent mating type loci HML and HMR, on $3 \mathrm{~L}$ and $3 \mathrm{R}$, respectively, are framed with a white rectangle. Sir3p is enriched in a small $1 \mathrm{~kb}$ region upstream of the TELACS at all telomeres. Repetitive and unmapped regions are shown in grey. The HML $\alpha$ reads have been eliminated as repetitive sequences during alignment to the reference genome which is MAT $\alpha$. C. Old (top left) and new (bottom left) Sir3p enrichment around TEL-ACS averaged for all 32 telomeres at indicated time points during the stationary and the renewed growth phases. The right panel shows average enrichment around the TEL-ACS for old and new Sir3p over time with Sir3p on and off rates during stationary phase and during the first cell cycle after release calculated from the slope of the linear fit as shown. D-E. Old and new Sir3p enrichment at HML (D) and HMR (E) at indicated time points (same color code for D and E) during the stationary and the growth phase after release. The right panel shows average enrichment over the entire silent mating type locus for old and new Sir3p over time with on and off rates as in $\mathrm{C}$. The time points marked replicate 1 and 2 come from two different time- course experiments.

Figure 2: Sir3p degradation rates increase after exit from stationary phase A.Western blot of old Sir3p-HA (anti-HA antibody) and new Sir3p-T7 (polyclonal anti T7 antibody) from total cell extracts during and after release from stationary phase. The top panel shows the experimental outline and describes the time points shown in the blot (marked by arrows above the blot). * marks a non-specific band detected with the anti-T7 antibody. The graph below the blot shows the quantification of the bands from the blot. Sir3p band intensities were first normalized to the a-tubulin loading control and then divided by the normalized Sir3p-HA intensity from mid-log cells (bar graph). The line plot inset shows the same normalized Sir3pHA and Sir3p-T7 band intensities for all time points after mid-log. B. Sir3p half life in stationary phase and after exit from stationary phase. The top plot shows the decrease in 
Sir3HA band intensity over time from the western blot. Since Sir3 mRNA synthesis is negligible in stationary phase, the half-life of Sir3p in stationary phase half-lives can be calculated directly from the decrease in Sir3-HA band intensity even though the tag switch was completed after $16 \mathrm{hrs}$, according to the equation: HLs=-b/(2*a), where $\mathrm{b}$ and $\mathrm{a}$ are the $\mathrm{y}$ cutoff and slope of the stationary phase linear fit equation (green line). The half-life of Sir3p after release HLg is calculated with the same equation using the y-cutoff and slope from the linear fit in the bottom plot.

Figure 3: The SIR complex is destabilized upon exit from stationary phase. A. Aminoacid sequence alignment of the C-terminus of Sir3 with differente epitope tags used in this study. The Sir3 strain (WT1) is the parent strain of all the RITE Sir3 tagged strains below.The Sir3-3xHA strain The Sir3-3xHA is the RITE strain used throughout the study. The tag switch was not induced in this experiment. All the strains marked with "after tag switch" were selected from their respective RITE strains (the strain listed in the line above the "after the tag switch strain") after the tag switch was completed. The Sir3-3xHA (-His tag) is another RITE strain with a Sir3-3xHA to $3 \times \mathrm{xT7}$ switch that does not have a $6 \mathrm{xHis}$ linker between the Cterminus and the first LoxP site. B. $\alpha$-factor heterochromatin stability test. The diagram on top shows the expected response of MATa cells to $\alpha$-factor added after release from stationary phase. If the SIR heterochromatic complex is unstable HML $\alpha$ and HMRa will be transcribed along with MATa, thus creating pseudo-diploid cells that don't respond to $\alpha$-factor and consequently do not become shmoo but start budding instead. The images show examples of frames from a live cell imaging experiment that follows Sir3-3xHA, Sir3-3xT7 and Sir3 (WT1) log phase cells or stationary phase cells in the first cell cycle after release in the presence of $0.2 \mu \mathrm{g} / \mathrm{ml} \alpha$-factor 60 and $420 \mathrm{~min}$ after the beginning of the time course. Red arrows: budding cells; White arrows: shmoos. C; The bar graph shows the fraction of budding cells out of the total number of cells (budding and shmoo) for each strain and growth condition from $\mathrm{B}$. The error bars are the standard deviation from the mean of all the replicates summarized in the table below the graph. D. same as $\mathrm{C}$ for the remaining set of strains from $\mathrm{A}$ that were not shown in B-C. E. $\alpha$-factor test with the OE Sir3p strain which shmoos independently of Sir3p in the presence of $\alpha$-factor because of the deletion of HML and MAT loci. F. Sir3 gene expression. Median Sir3 read density enrichment from the RNA-seq dataset from Figure 6 normalized to an external spike-in S.pombe RNA control.

Figure 4: A. Diagram of the Sir3p gene construct controlled by a Galactose inducible promoter in the Sir3p OE strain (OverExpression) (top left). Bottom: Experiment outline. Cells were arrested by galactose depletion, and released of fresh media with the indicated carbon source (2\%) and allowed to grow for 2 and 12 doublings (monitored by OD measurements). Cell aliquots were fixed with $1 \%$ formaldehyde for $20 \mathrm{~min}$ at times indicated 
below the diagrams and anti-HA ChIPs were performed on sonicated chromatin. B. Heat map of Sir3p (HA tag) enrichment over input during and after exit from quiescence, at all yeast telomeres (30kbp from chromosomes ends). Time points are aligned by the ARS Consensus Sequence (TEL-ACS) located in telomeric silencer regions, which are Sir complex nucleation sites at telomeres. Silent mating type loci HML (HML is deleted in this strain) and HMR, on $3 \mathrm{~L}$ and $3 \mathrm{R}$, respectively, are framed with a white rectangle. Repetitive and unmapped regions are shown in grey. C-E. Sir3p enrichment around TEL-ACS averaged for all 32 telomeres after release into Galactose- over expression of Sir3p (C), Dextrose- inhibition of Sir3p expression (D) or Raffinose- low Sir3p expression (E). F. Average Sir3p enrichment around the TEL-ACS over time in indicated carbon sources. G-I. Sir3p enrichment at HMR after release into Galactose (G), Dextrose (H) or Raffinose (I). J. Average Sir3p enrichment over the entire HMR over time .

Figure 5: Apparent Sir3 dependent transient upregulation of subtelomeric genes after exit from stationary phase. A. Left: Genotypes of yeast strains used in the RT and anti-HA Sir3p-HA ChIP qPCR outlined on the right. B. RT-qPCR of subtelomeric genes. Bar plot of normalized $\mathrm{Ct}$ values (Ct(SSL2)-Ct) for 6 subtelomeric genes and HMRa2. Cts were normalized by subtraction from the $\mathrm{Ct}$ of the non subtelomeric gene SSL2 in each time point and strain (the values in the graph are an average of three technical replicates with 1x, 10x and 100x cDNA dilutions). PAU genes are highly repetitive and our primers bind multiple PAU targets as indicated above the plots. Likewise the HMRa2 primers also bind to HMLalpha2 and MATa2. Note that HMRa2 expression in the WT strains appears higher than in the oeSir3 strain because our primers also bind to MATa2, which is expressed in both WT strains but not in the oeSiR3 strain where the MAT locus is deleted. C. RT-QPCR (experiment from B) and Sir3p ChIP-qPCR (average of 2 replicates) of 6 subtelomeric genes and HMRa2 and the non subtelomeric SSL2 control for oeSir3 and Sir3-3xHA strains. Cts from RT-QPCR were normalized as in B with the SSL2 negative control. Cts from Sir3p ChIPs and their corresponding Inputs qPCR reactions from Sir3-3xHA, oeSir3 and Sir3 $\Delta$ strains were normalized to the average $\mathrm{Ct}$ of three genes used as negative internal controls: YGL177W, YGL188C and YLR112W, to obtain $\Delta \mathrm{Ct}$. Each Input $\Delta \mathrm{Ct}$ was then subtracted from its corresponding ChIP $\Delta \mathrm{Ct}$ to get $\Delta \Delta \mathrm{Ct}$. $\Delta \Delta \mathrm{Cts}$ from the Sir3 $\Delta$ strain were subtracted from the $\Delta \Delta$ Cts from oeSir3 and wt Sir3p strains to eliminate Sir3p non-specific signals. The ChIP-qPCR values in the graph are an average of two biological replicates with the magenta shaded area representing the standard deviation. 
Figure 6: Genome-wide transcription is reactivated faster after exit from stationary phase when SIR function is compromised. A. RNA-seq libraries were made from the same RNA samples that were used in Figure 5. Heat map of median mRNA enrichment (normalized to an external spike in control of S.pombe RNA) in indicated strains before and after release from stationary phase for $\sim 5800$ yeast genes. Genes were grouped into four clusters based on average mRNA abundance in WT1 cells (bar graph on the left). The pearson correlation between the time course expression profile in each strain and the WT1 strain was determined for each gene. Genes in each cluster were then ordered by increasing difference between the Sir3 $\Delta$ correlation to WT1 and the W2 correlation to WT1 ( $\Delta$ correlation(Sir3 $\Delta$ WT2)). The gene quartile within each cluster with the most negative $\Delta$ correlation(Sir3 $\Delta$ WT2) was considered to contain genes whose expression profiles between $\operatorname{Sir} 3 \Delta$ and WT cells were the least similar (marked in black). The gene quartile within each cluster with the $\Delta$ correlation(Sir3 $\Delta$-WT2) closest to 0 was considered to contain genes whose expression profiles between $\operatorname{Sir} 3 \Delta$ and WT cells were the most similar (marked in red) B. Gene expression profiles were averaged for most and least similar gene quartiles in each cluster. The average profile from each strain indicated in the strip header on the left (blue) is compared to the average expression profile of WT1 (magenta). 
bioRxiv preprint doi: https://doi.org/10.1101/603613; this version posted March 22, 2021. The copyright holder for this preprint (which was not certified by peer review) is the author/funder. All rights reserved. No reuse allowed without permission.

A (s288C: MATa, HML $\alpha, H M R a, \triangle b a r 1, p(H I S 3$ Pgpd_CRE_EBD78))
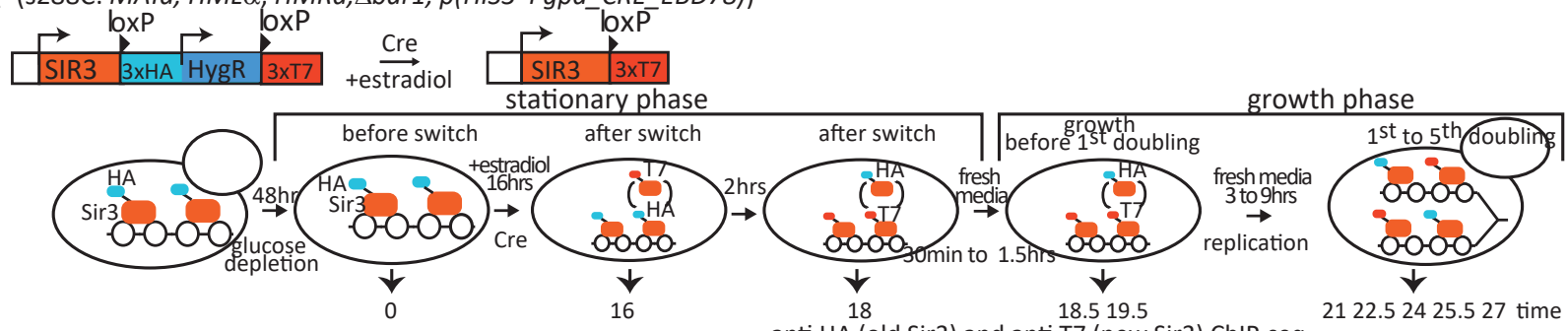

anti HA (old Sir3) and anti T7 (new Sir3) ChIP-seq

2122.52425 .527 time points (hrs)

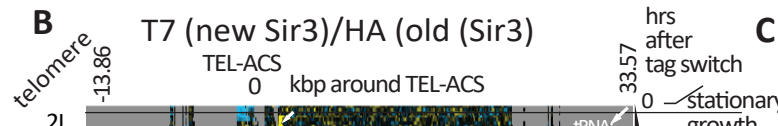

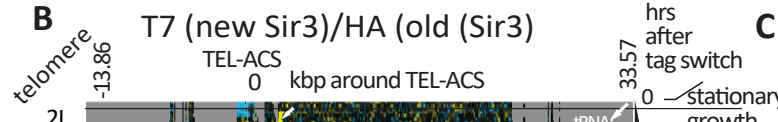

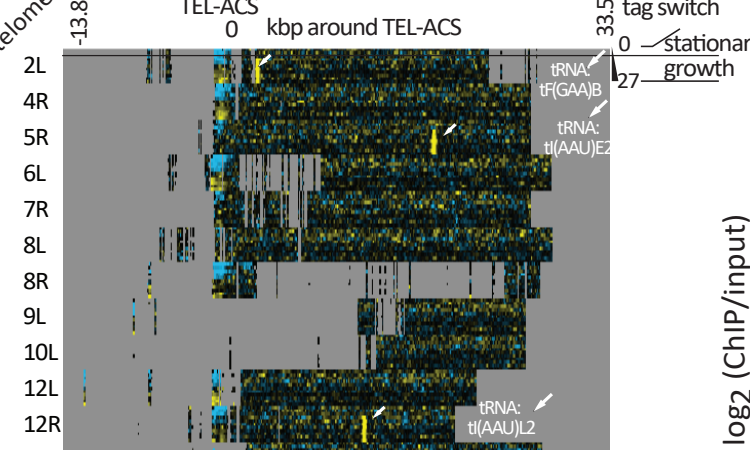

$13 \mathrm{~L}$

$14 \mathrm{~L}$

$15 \mathrm{R}$

$5 \mathrm{~L}$

$16 \mathrm{R}$

$1 R$
$6 R$

$6 R$

$2 \mathrm{R}$

$2 \mathrm{R}$
$3 \mathrm{~L}$

3R

4L

$7 \mathrm{~L}$
$9 \mathrm{R}$

$9 \mathrm{R}$
$10 \mathrm{R}$

$11 \mathrm{~L}$

$11 R$
$13 R$

$14 \mathrm{R}$

$15 \mathrm{~L}$

C anti HA (old Sir3)
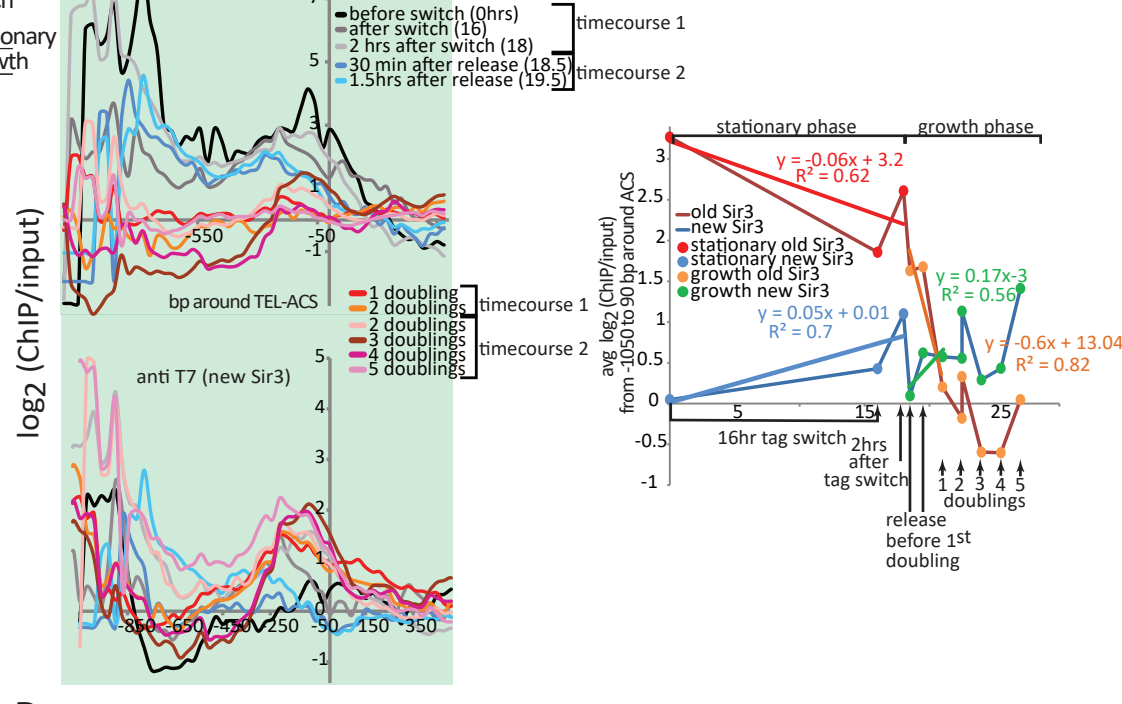

tit i

Iiliniti

HML

14 III

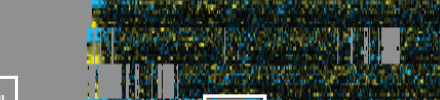

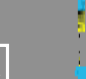

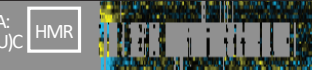
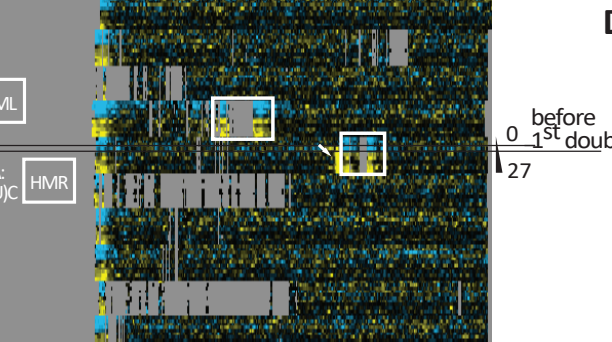

D
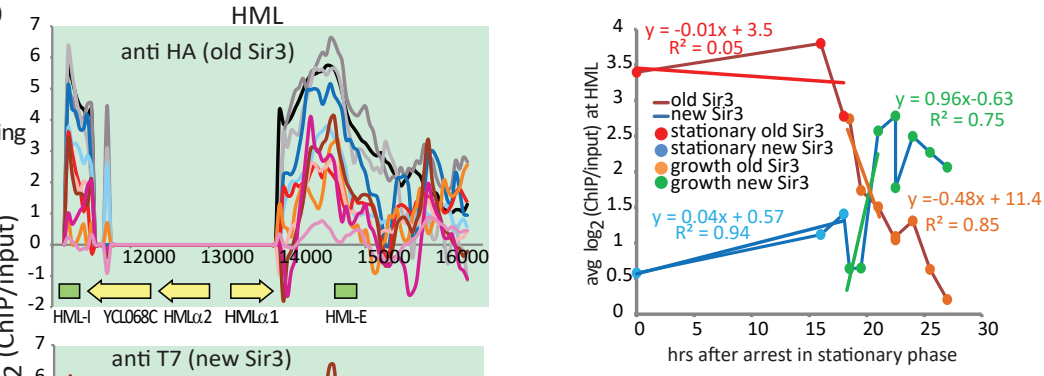

过
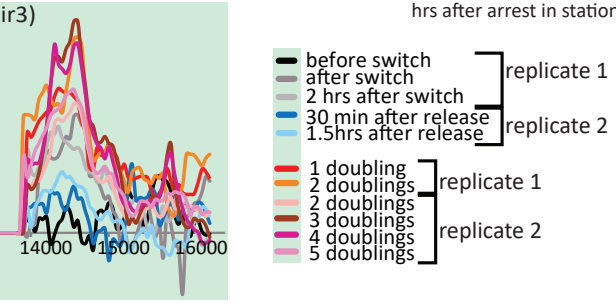

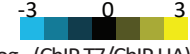

$\log _{2}$ (ChIP T7/ChIP HA)
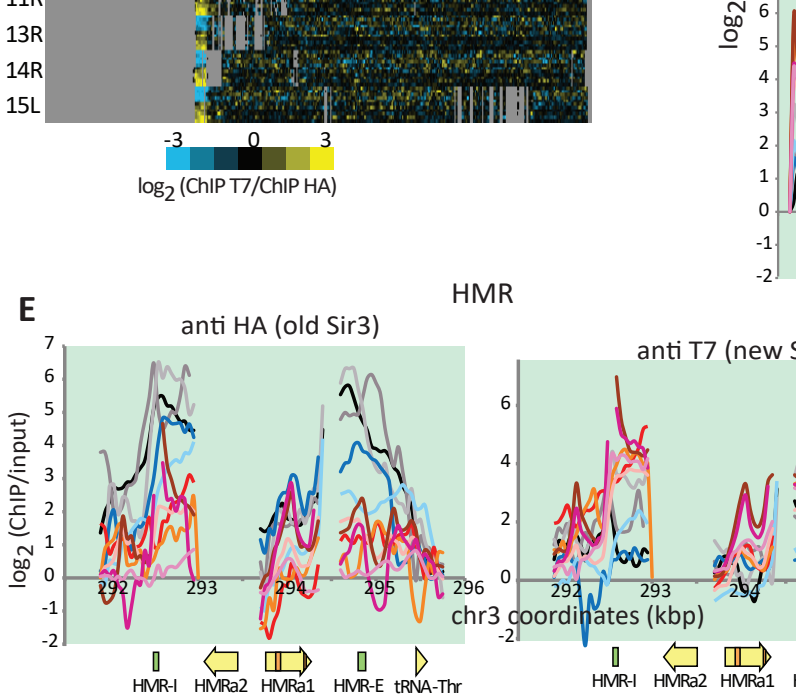

HMR

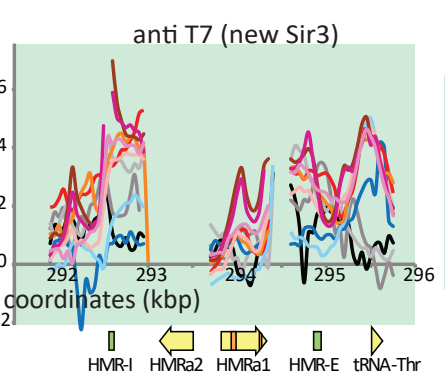

ordinates (bp) 
A

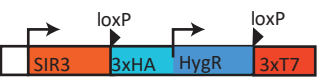

$\underset{+ \text { estradiol }}{\stackrel{\text { Cre }}{\longrightarrow}}$

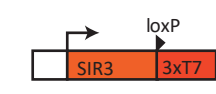

growth phase

0.16

0.16
0.14 $\quad \begin{gathered}y=-0.0025 x+0.14 \\ R^{2}=1\end{gathered}$

growth phase

sationary phase

log phase stationary phase s: $:$ /agisorg/stow Sir3HA

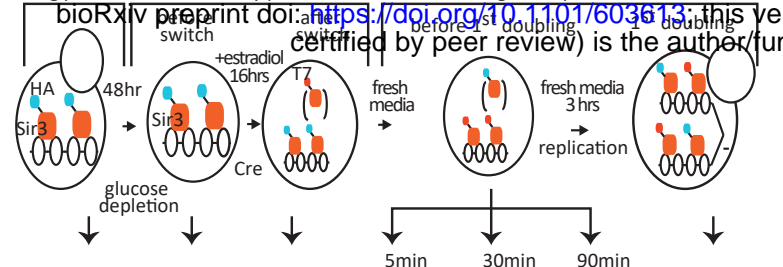

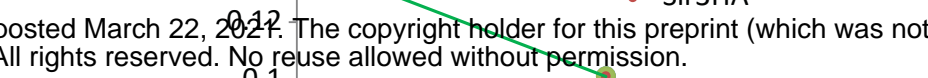

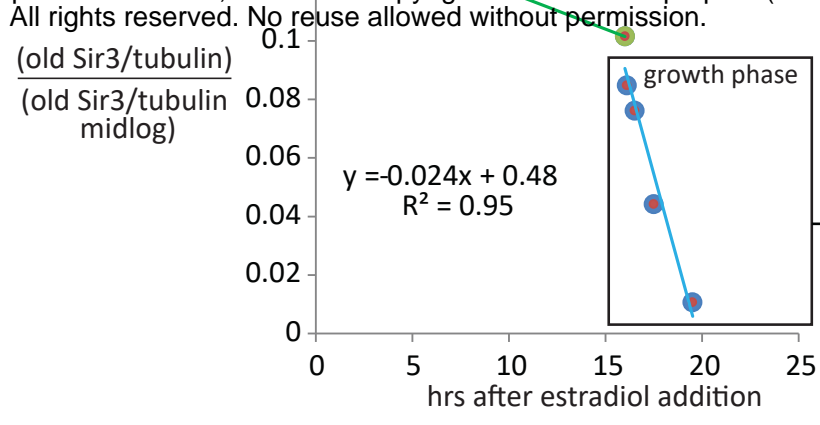

$H L_{s}=-0.14 /(-0.0025 * 2)=28 \mathrm{hrs}$

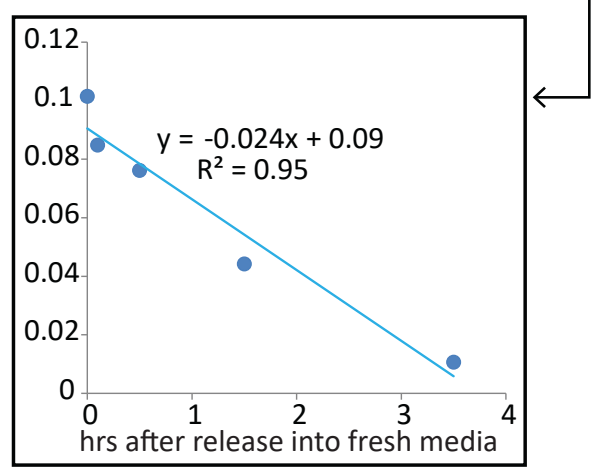

$H L_{g}=60 \mathrm{~min} *(-0.09 /(-0.024 * 2))=112.5 \mathrm{~min}$

Figure 2: Sir3p degradation rate increases 10 fold upon release from stationary phase 
A $\operatorname{sir} 3$ (WT1)

998

1018

1028

1038

1048

\section{Sir3-3xHA}

MDEEFKNMDC I

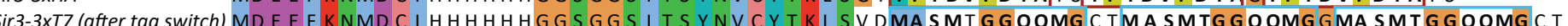

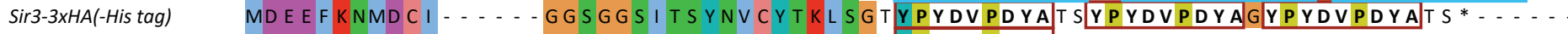

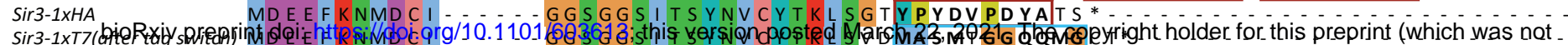

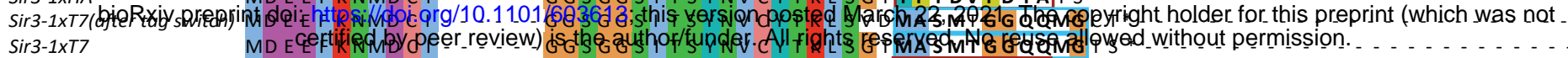
Sir3-1XHA(after tag switch) MDE E F KNMD C I . . . . G G S G G I T S Y NV CY T KL SVDYPYDVPDYA C * . . . . . . . . . . . . . . . .

B

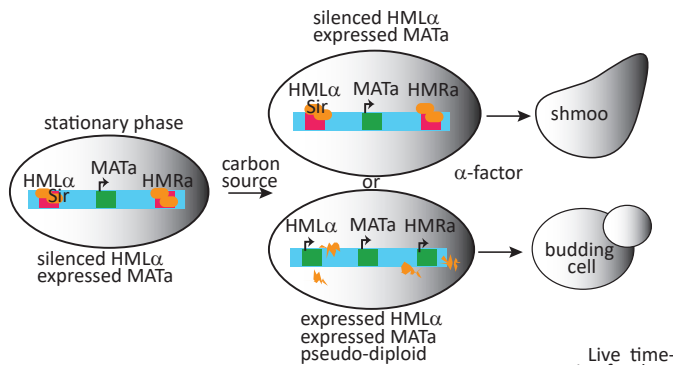

(s288C: MATa, HML $\alpha, H M R a, \triangle b a r 1, p(H I S 3$ Pgpd_CRE_EBD78)) Sir3 3 Sir3 $3 \times$ HA HygR $3 \times T 7$ SIR3-3xHA

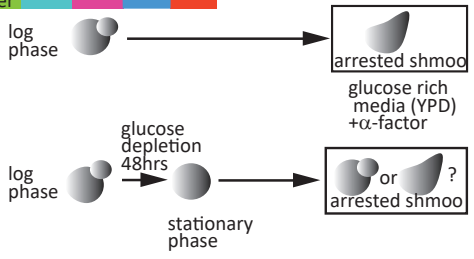

(s288C: MATa, HML $\alpha, H M R a, \triangle b a r 1, p(H I S 3$ Pgpd_CRE_EBD78))

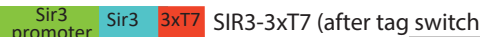

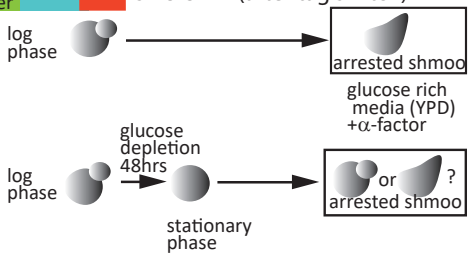

(s288C: MATa, HML $\alpha, H M R a, \triangle b a r 1, p(H I S 3$ Pgpd_CRE_EBD78)) Sir3
promoter

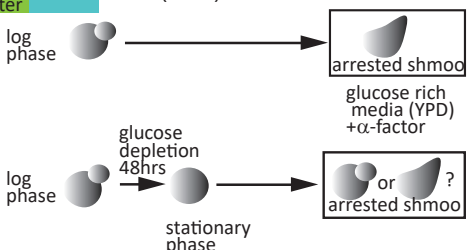

Live time-lapse microscopy, 60
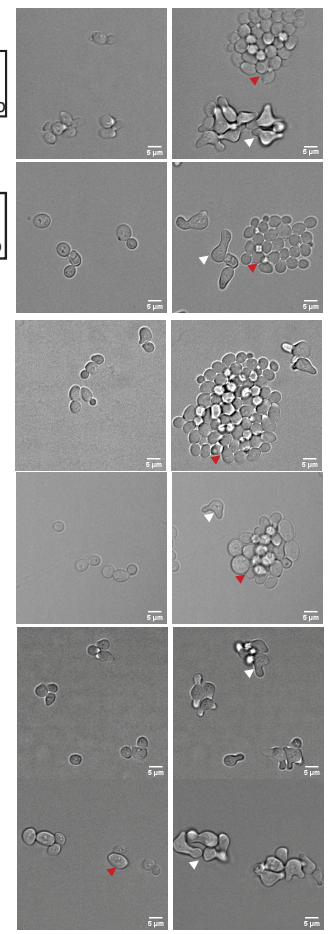

$E$

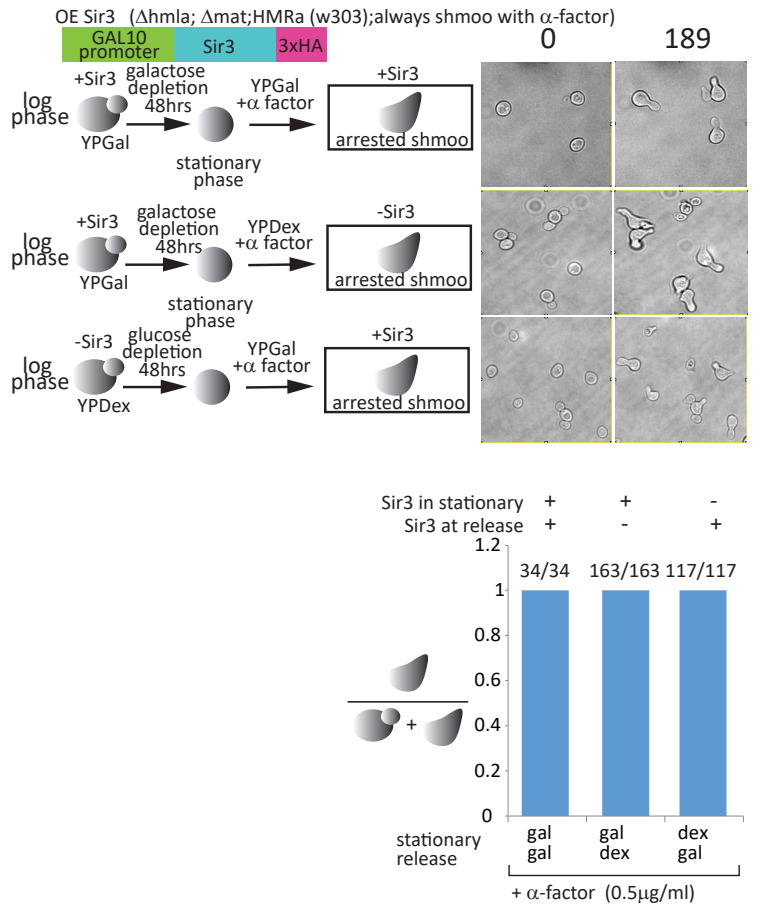

C

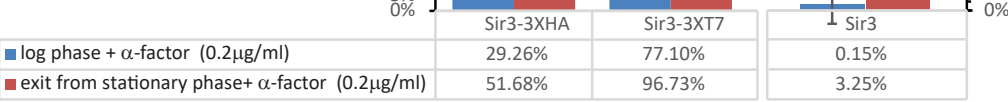

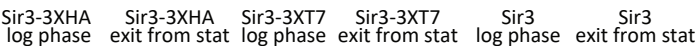

\begin{tabular}{lllllll}
$\mathrm{n}$ replicates & 11 & 9 & 4 & 4 & 8 & 8 \\
\hline total $\mathrm{n}$ cells & 5806 & 3436 & 2437 & 1787 & 6707 & 3234 \\
\hline total $\mathrm{n}$ dividing cells & 1755 & 1801 & 1758 & 1729 & 10 & 105 \\
\hline
\end{tabular}

D
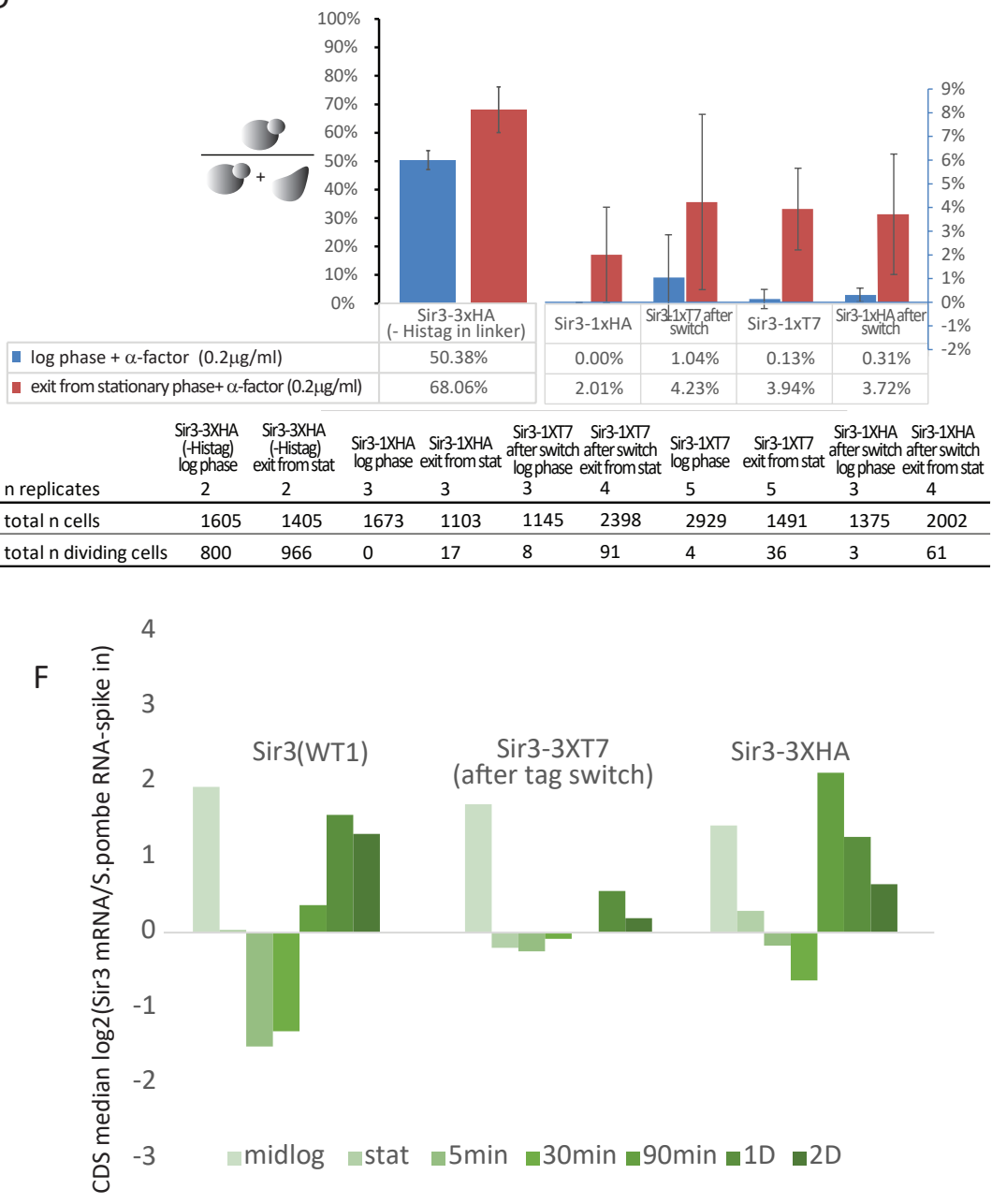

$-4$

Figure 3: Cells are less sensitive to alpha-factor upon exit from stationary phase. 
bioRxiv preprint doi: https://doi.org/10.1101/603613; this version posted March 22, 2021. The copyright holder for this preprint (which was not certified by peer review) is the author/funder. All rights reserved. No reuse allowed without permission.

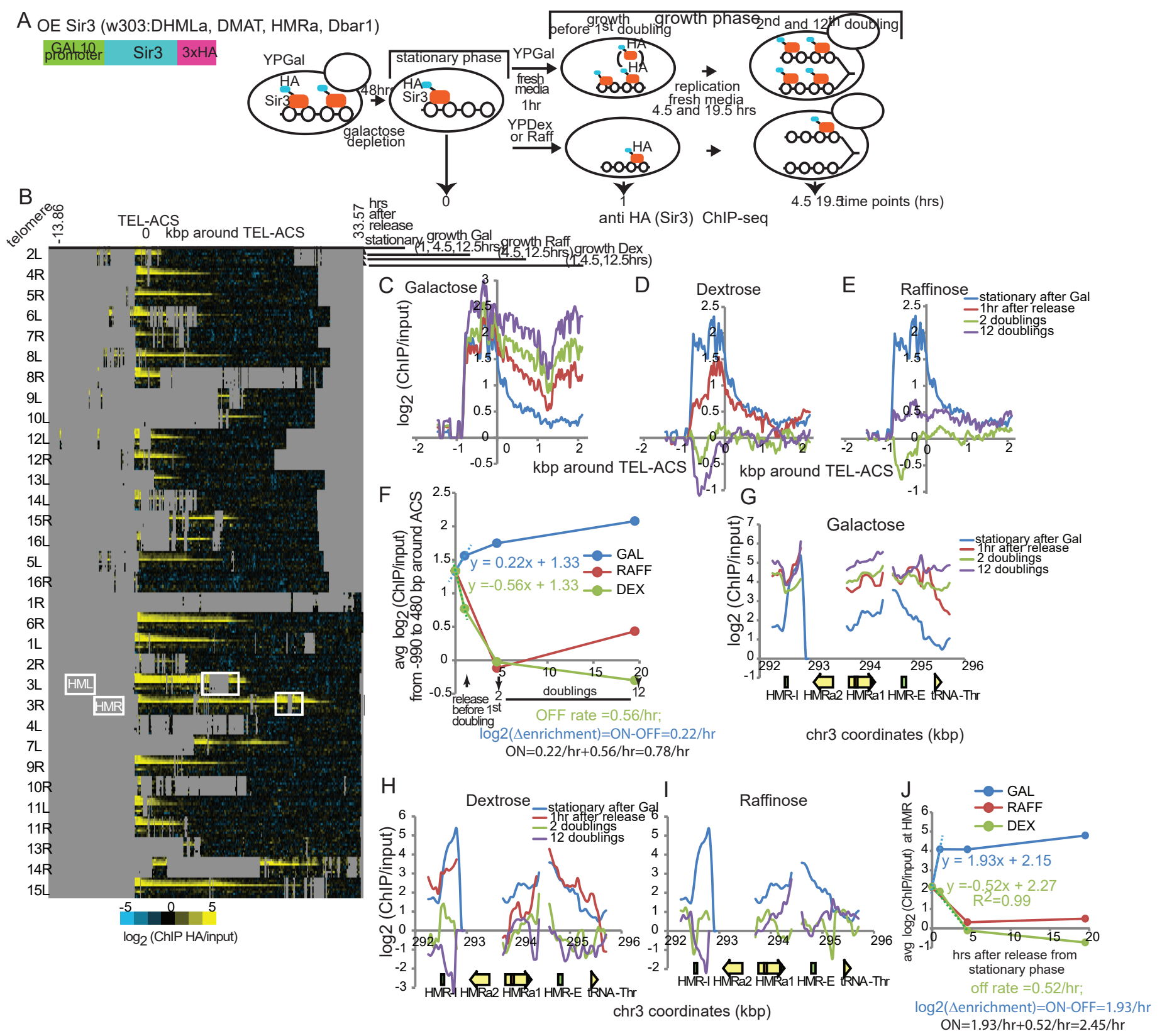

figure 4: Dynamics of Sir3 binding upon over expression of Sir3 during exit from stationary phase 
A (s288C:MATa, HML $\alpha$, HMRa,_Lar1, p(HIS3 Pgpd_CRE_EBD78))

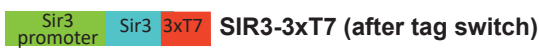
(s288C:MATa, HMLa, HMRa,_Lbar1, p(HIS3 Pgpd_CRE_EBD78)) Sir3
promoter Sir3 $3 \times$ HA HygR $3 \times T 7$ SIR3-3xHA

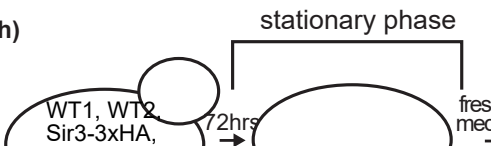

growth phase
before 1 1Sth doubling

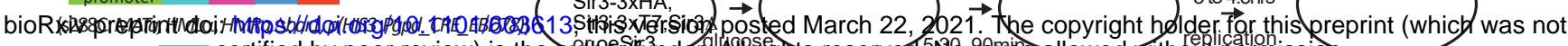

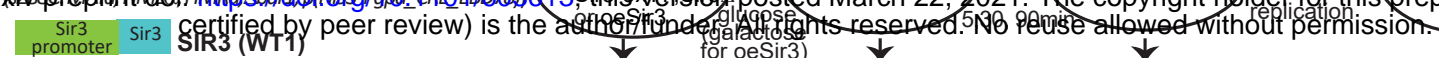

(s288C: MATa, HML $\alpha, H M R a, \Delta b a r 1$ )

Sir3 Sir3 SIR3 (WT2)

$\underset{\text { midlog }}{\downarrow} \begin{gathered}\text { for oesir3) } \\ \text { depletion }\end{gathered}$

$5,0.5(30)$ and $1.5(90)$ hrs (min) $3.5 \mathrm{hrs}(1 \mathrm{D})$ and $5 \mathrm{hrs}(2 \mathrm{D})$ time points

( $\Delta$ hmla; $\triangle$ mat;HMRa (w303))

GAL10
promoter Sir3 $3 \times H A$ OE Sir3

B
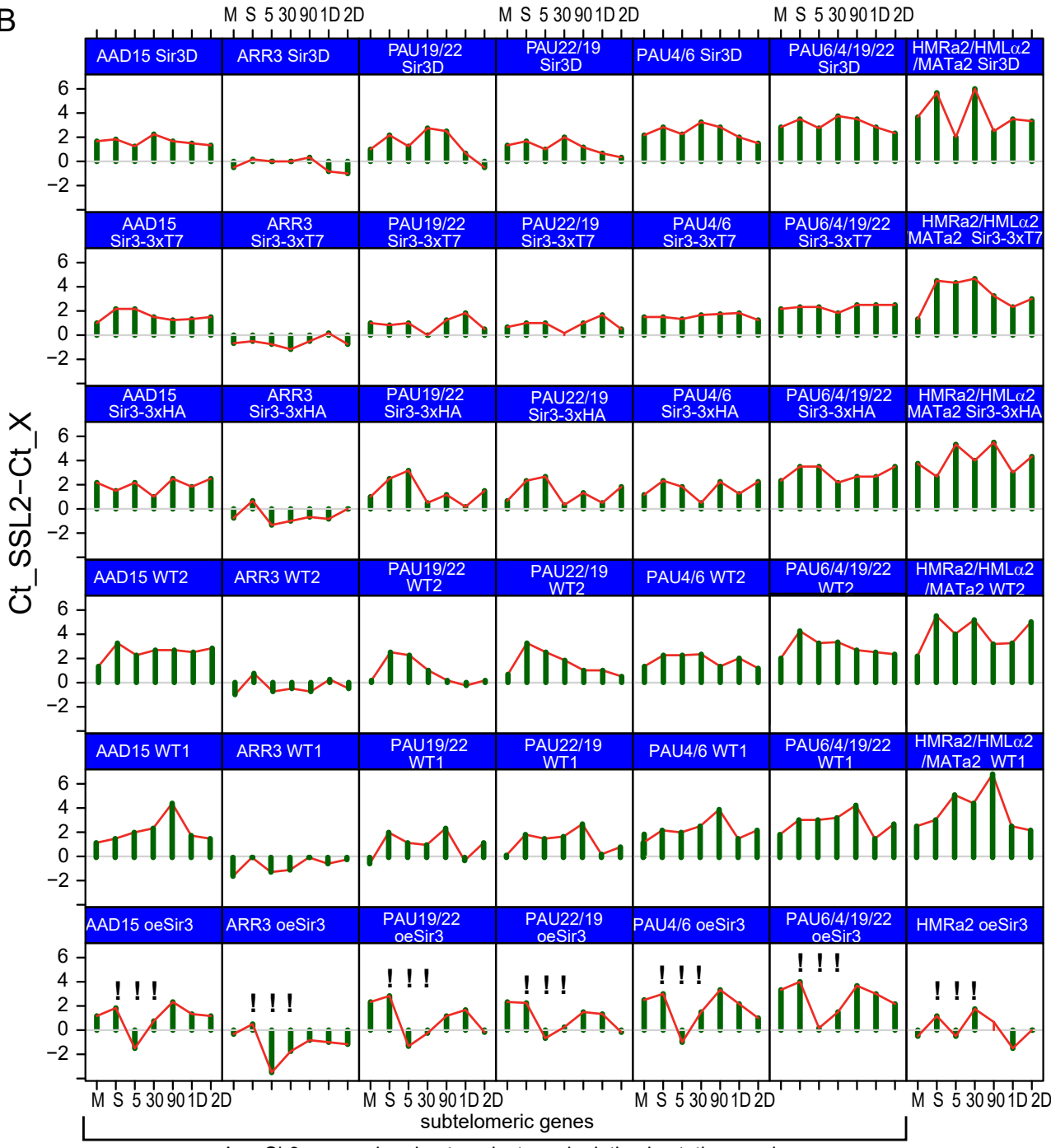

M: midlog;

S: Stationary phase;

5, 30, 90: 30 and $90 \mathrm{~min}$ after release;

1D,2D: $1^{\text {st }}$ and $2^{\text {nd }}$ division after release

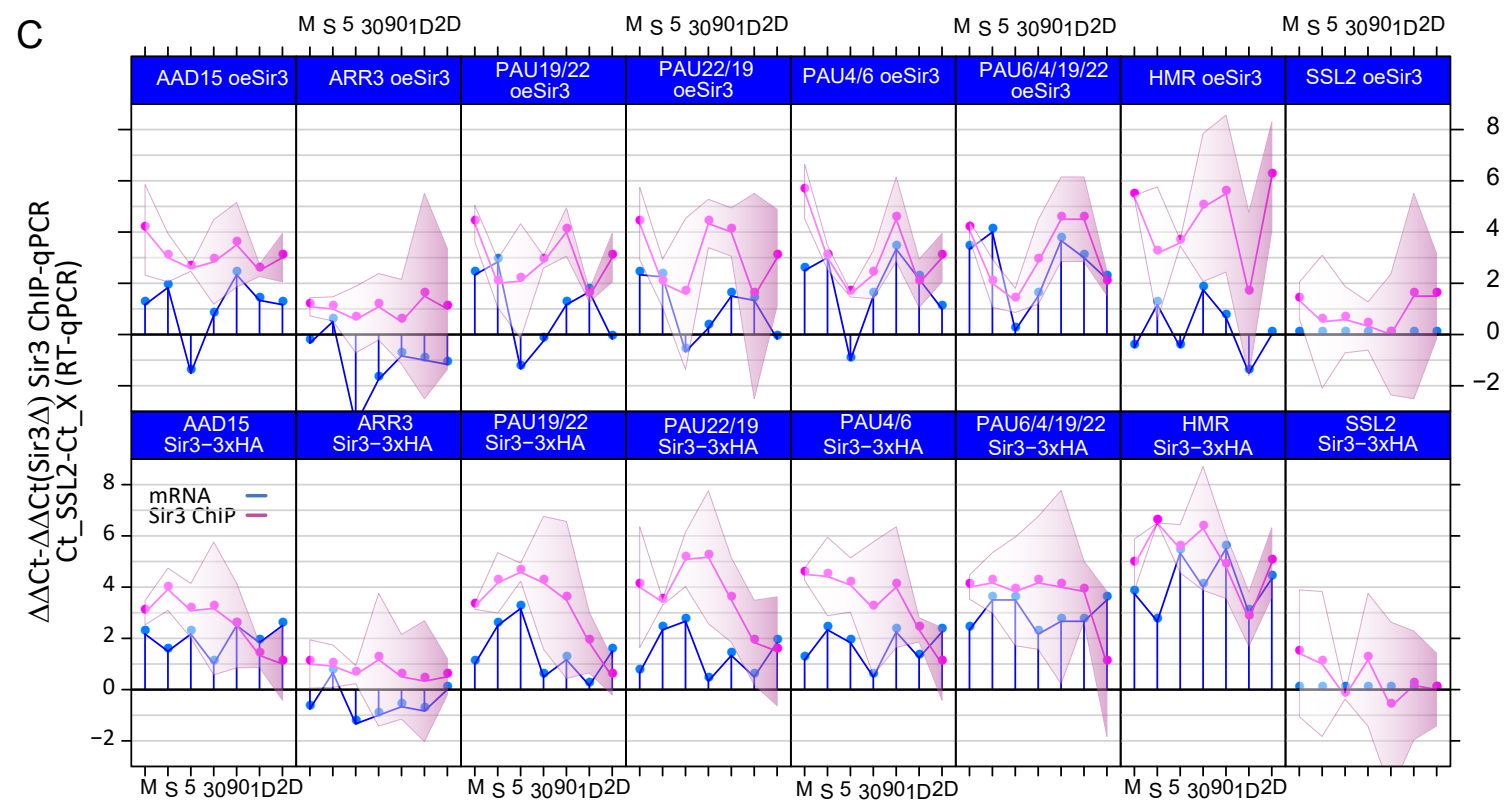

Figure 5: Subtelomeric genes and HMRa2 are transiently upregulated in WT cells upon exit from stationary phase 


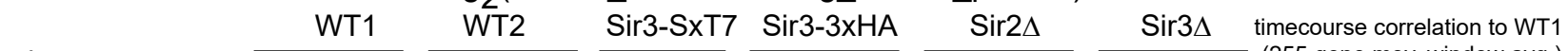

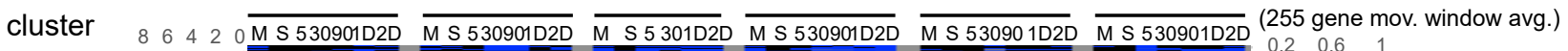

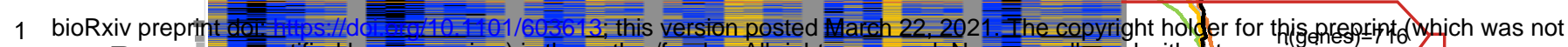

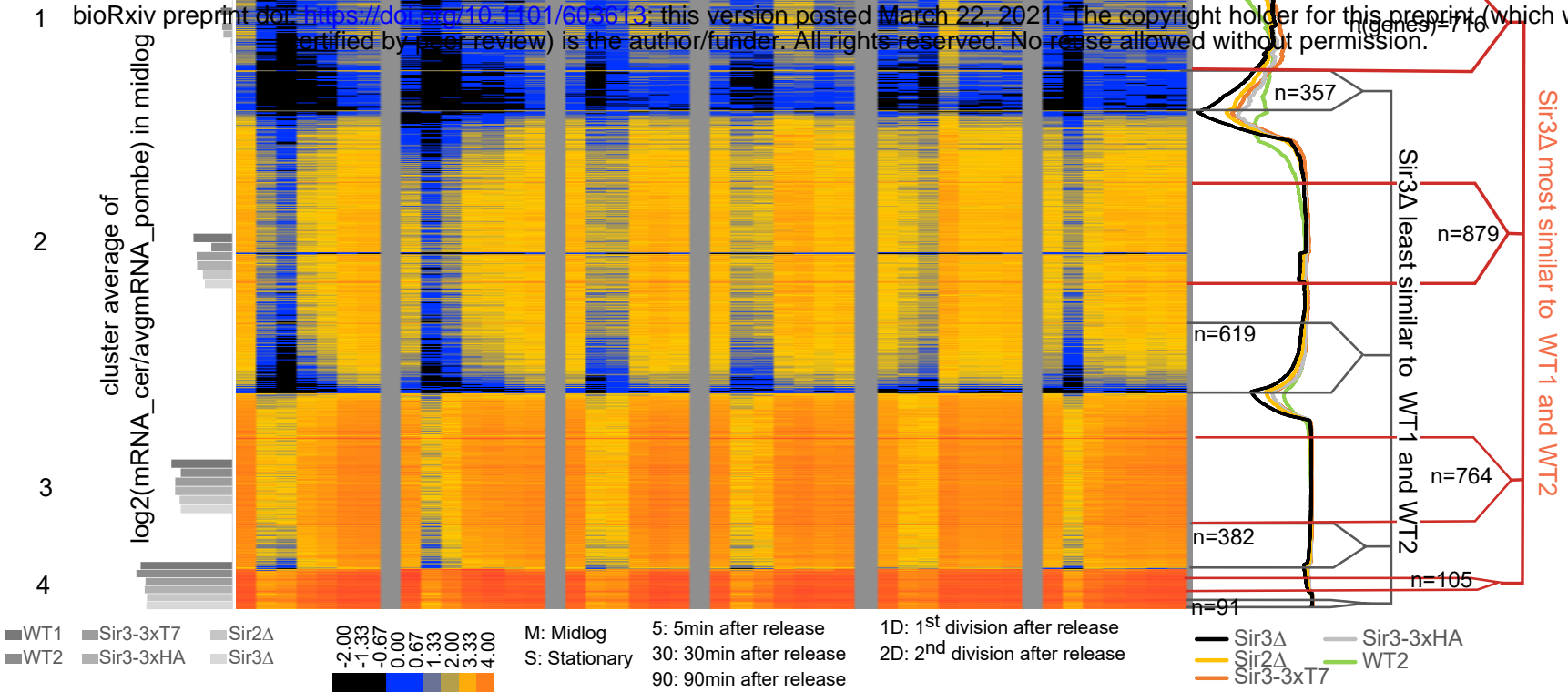

B
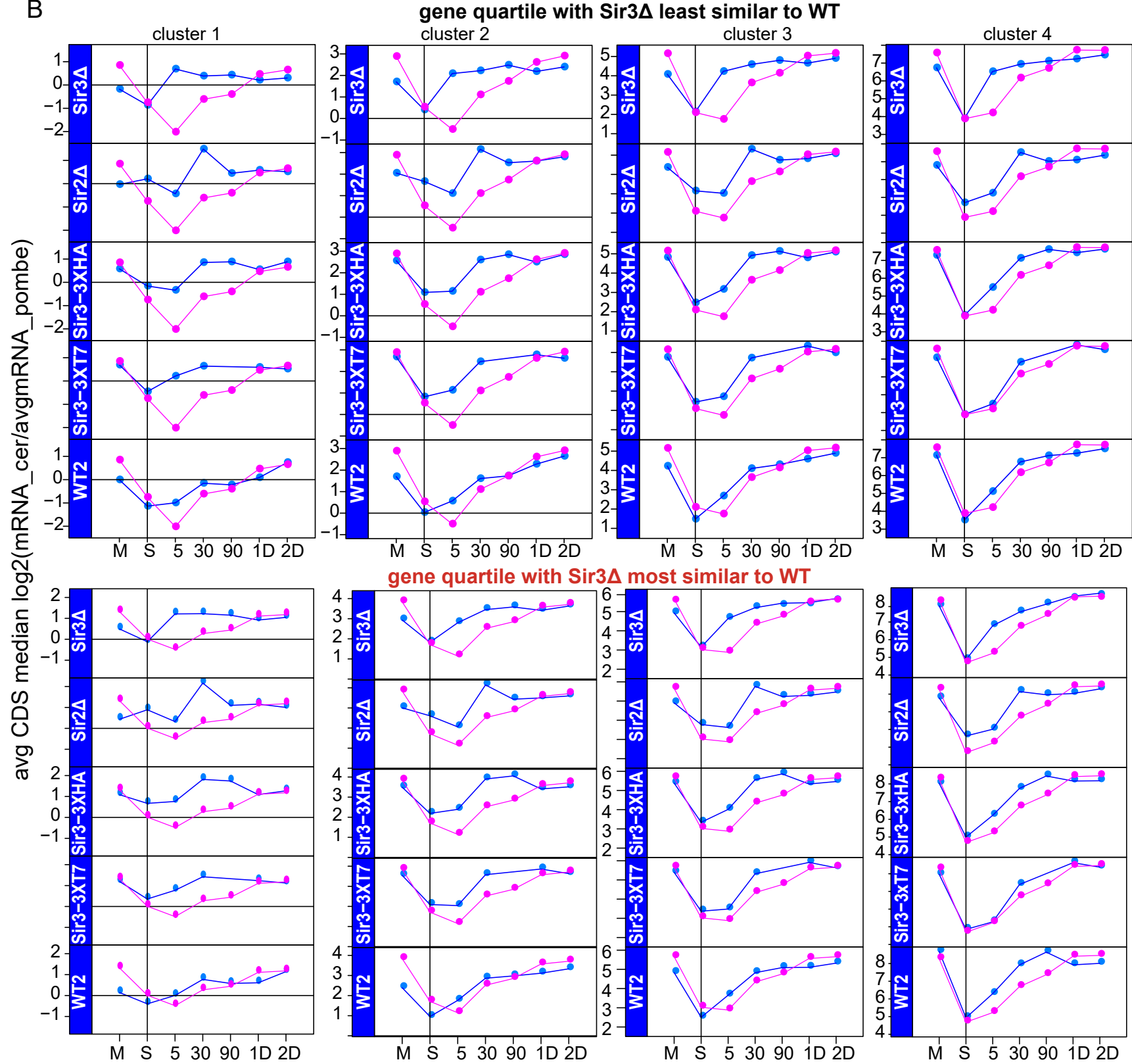

WT1

Figure 6: RNA-seq time course after exit from stationary phase normalized with "spiked in" S.pombe RNA 
A (s288C: Mnt $\rightarrow \quad$ loxP $\rightarrow \quad$ lOXPcertified by peer reyiw) is phe Pauthor/funder. All rights reserved. No reuse allowed without permission.

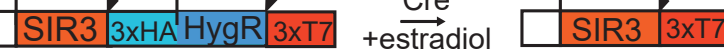

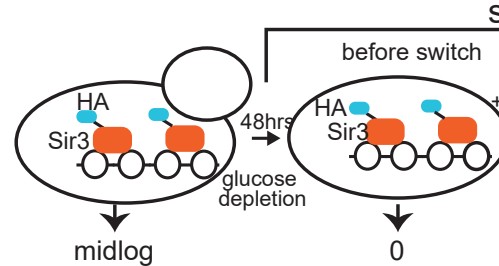

stationary phase

growth phase

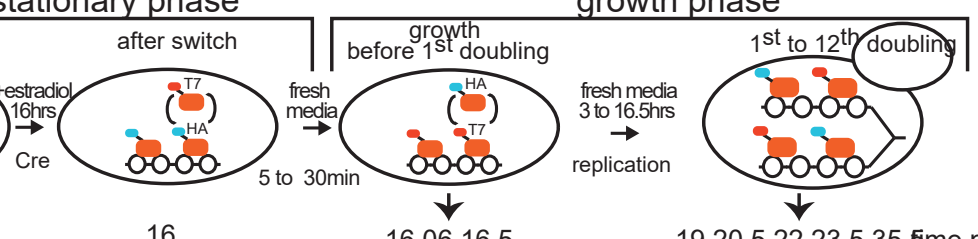

anti HA (old Sir3) and anti T7 (new Sir3) ChIP-seq

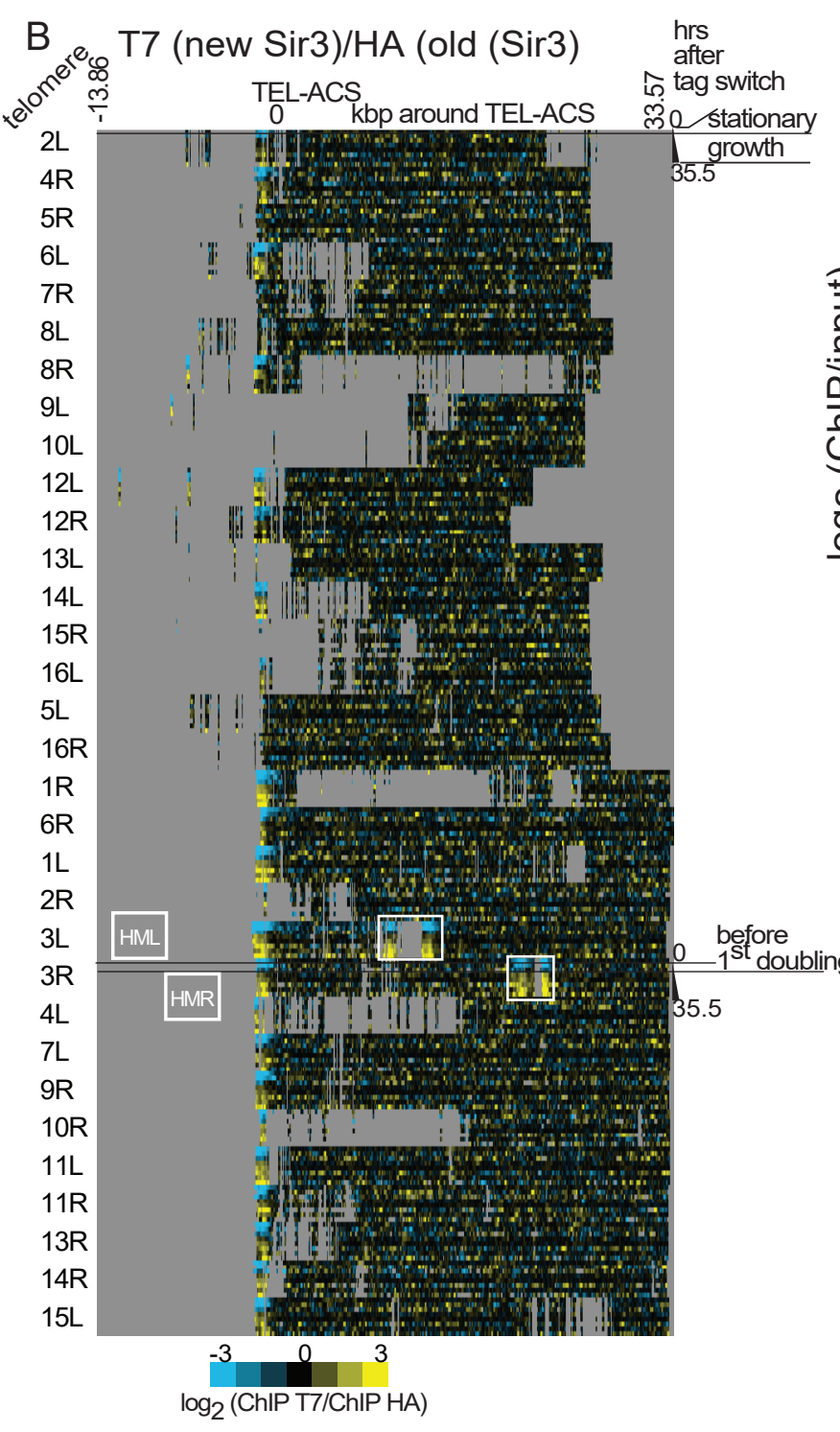

HML

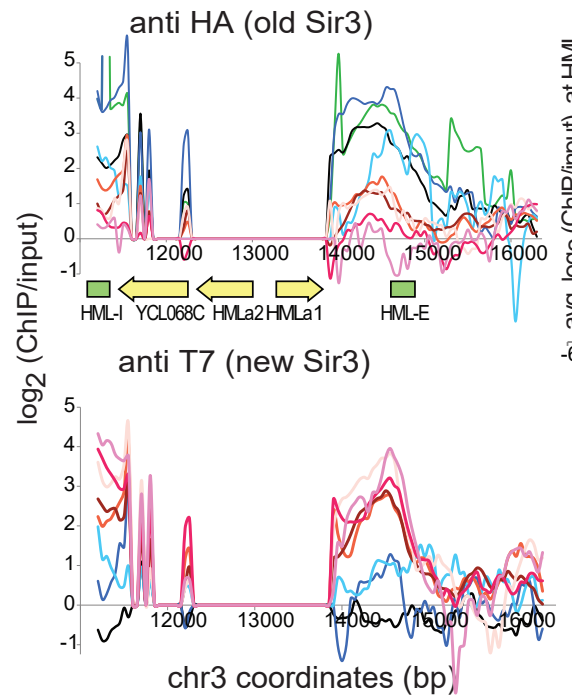

chr3 coordinates (bp)

anti HA (old Sir3) $\left.{ }^{5}\right] \quad$ =before $_{\text {bwitch }}$
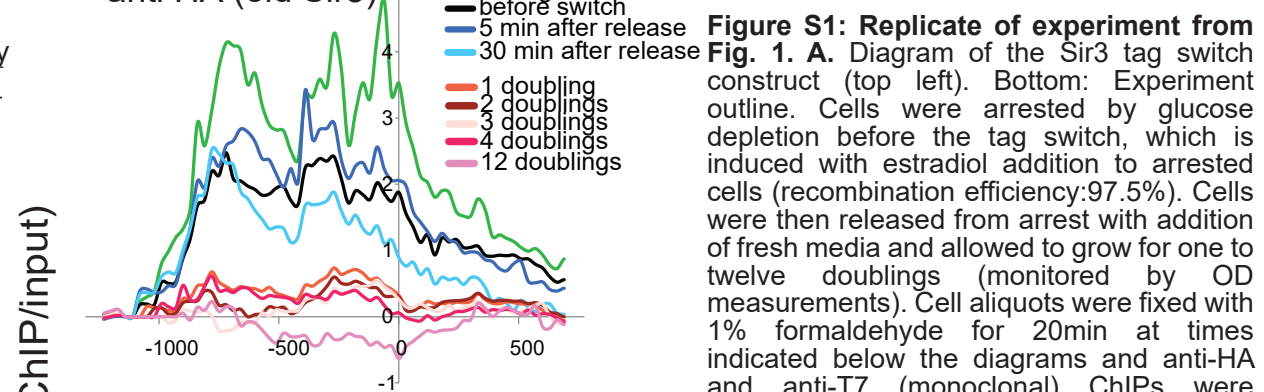

anti T7 (new Sir3)

으 으

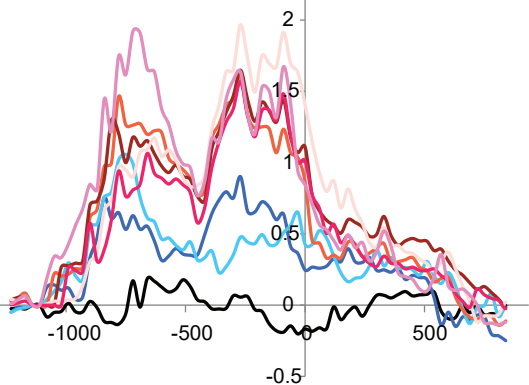

bp around TEL-ACS

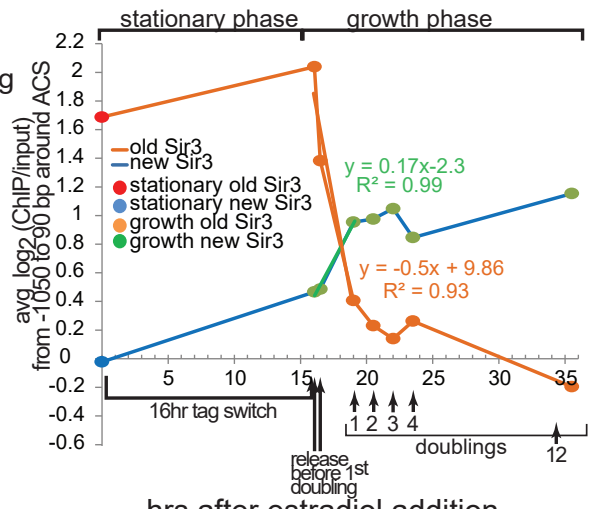

hrs after estradiol addition

E
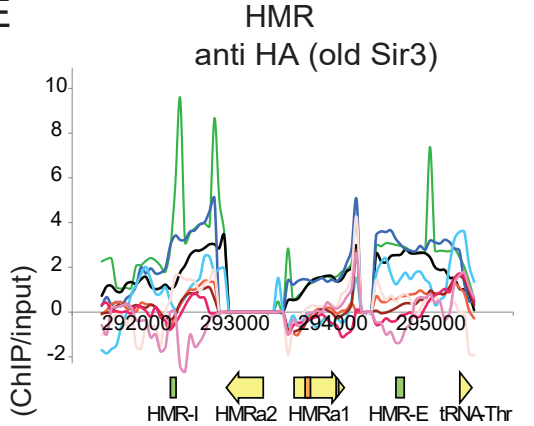

잉 7

hrs after arrest in stationary phase
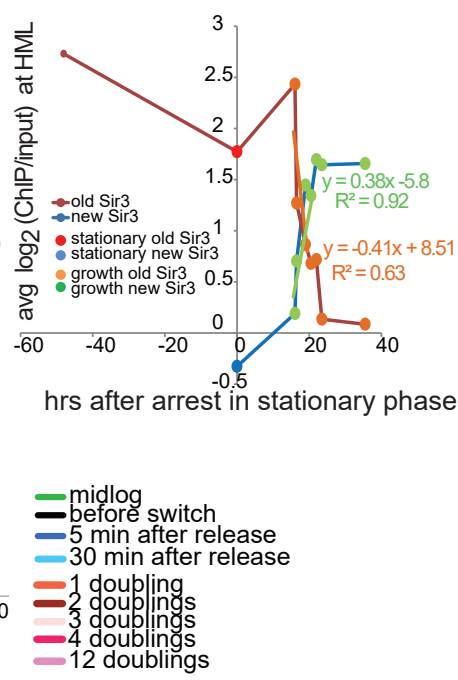
construct (top left). Bottom: Experiment outline. Cells were arrested by glucose depletion before the tag switch, which is induced with estradiol addition to arrested
cells (recombination efficiency: $97.5 \%$ ). Cells were then released from arrest with addition of fresh media and allowed to grow for one to twelve doublings (monitored by OD measurements). Cell aliquots were fixed with $1 \%$ formaldehyde for $20 \mathrm{~min}$ at times indicated below the diagrams and anti-HA and anti-T7 (monoclonal) ChIPs were performed on sonicated chromatin. B. Heat map of new Sir3 (T7 tag) enrichment over old Sir3 (HA tag) during and after exit from quiescence, at all yeast telomeres $(30 \mathrm{kbp}$ from chromosomes ends). Time points are aligned by the ARS Consensus Sequence (TEL-ACS) located in telomeric silencer regions i.e. Sir complex nucleation sites at telomeres. Silent mating type loci HML and $H M R$, on $3 L$ and $3 R$, respectively, are framed with a white rectangle. Sir3 is enriched in a small $1 \mathrm{~kb}$ region upstream of the TEL-ACS at all telomeres. Repetitive and unmapped regions are shown in grey. The HMLa reads have been eliminated as repetitive sequences during alignment to the reference genome which is MATa. C. Old (top) and new (middle) Sir3 enrichment around TEL-ACS averaged for all 32 telomeres at indicated time points during the stationary and the renewed growth phases. The bottom panel shows average enrichment around the TEL-ACS for old and new Sir3 over time with Sir3p on (green) and off (orange) rates during the first cycle after release calculated from slopes of linear fits of time points $5 \mathrm{~min}$, $30 \mathrm{~min}$ and 1 doubling after release as in Figure 1. D-E. Old (top left) and new (bottom left) Sir3 enrichment at HML (D) and HMR (E) at indicated time points (same color code for $\mathrm{D}$ and $\mathrm{E}$ ) during the stationary phase and the growth phase. after release. The right panel shows average enrichment over the entire silent mating type locus for old and new Sir3p over time.

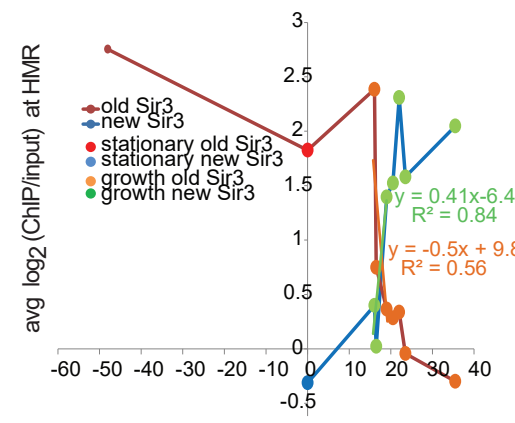

hrs after arrest in stationary phase 

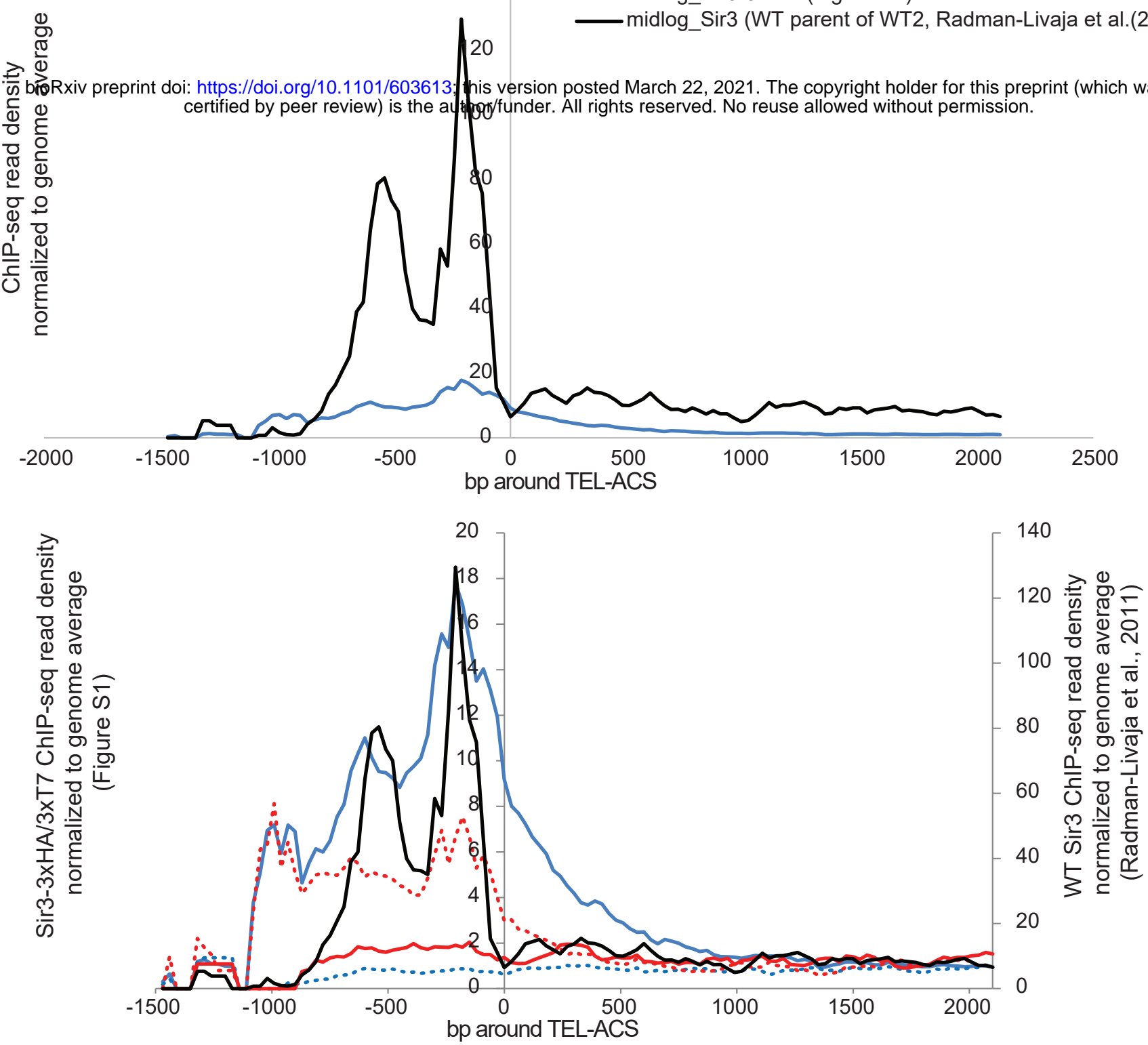

midlog_Sir3-3xHA (before tag switch) _ midlog_Sir3-3xT7 (before tag switch)

$\ldots . .12^{\text {th }}$ doubling_Sir3-3xHA (after tag switch) $\ldots . .12^{\text {th }}$ doubling_Sir3-3xT7 (after tag switch)

—_ midlog_Sir3 (WT parent of WT2, Radman-Livaja et al.(2011))

B

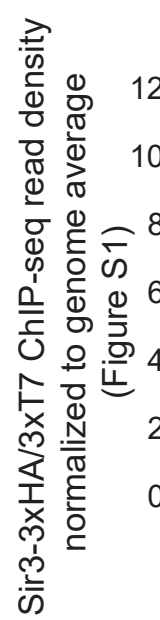

HMR 291

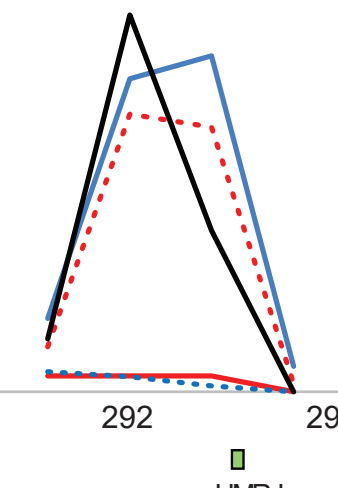

292

HMR-I

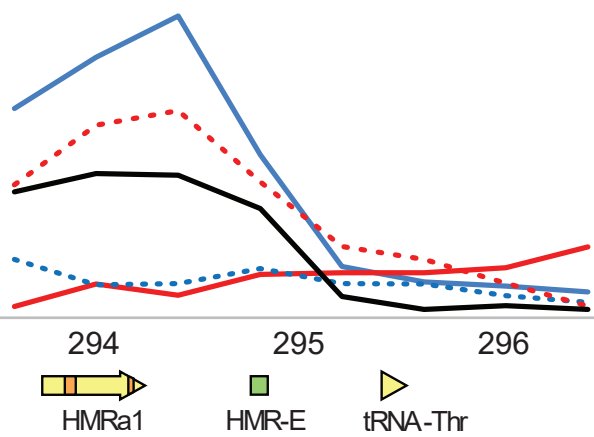

chr3 coordinates $(\mathrm{kbp})$

- midlog_Sir3-3xHA (before tag switch)

midlog_Sir3-3xT7 (before tag switch)

12 $2^{\text {th }}$ doubling_Sir3-3xHA (after tag switch) .12th doubling_Sir3-3xT7 (after tag switch)

—_ midlog_Sir3 (WT parent of WT2, Radman-Livaja et al.(2011))

Figure S2: Comparison of untagged and 3xHA/3xT7 tagged Sir3 ChIP-seq in subtelomeric regions $(\mathrm{A})$ and at the HMR silent mating type locus (B) 
bioRxiv preprint doi: https://doi.org/10.1101/603613; this version posted March 22, 2021. The copyright holder for this preprint (which was not
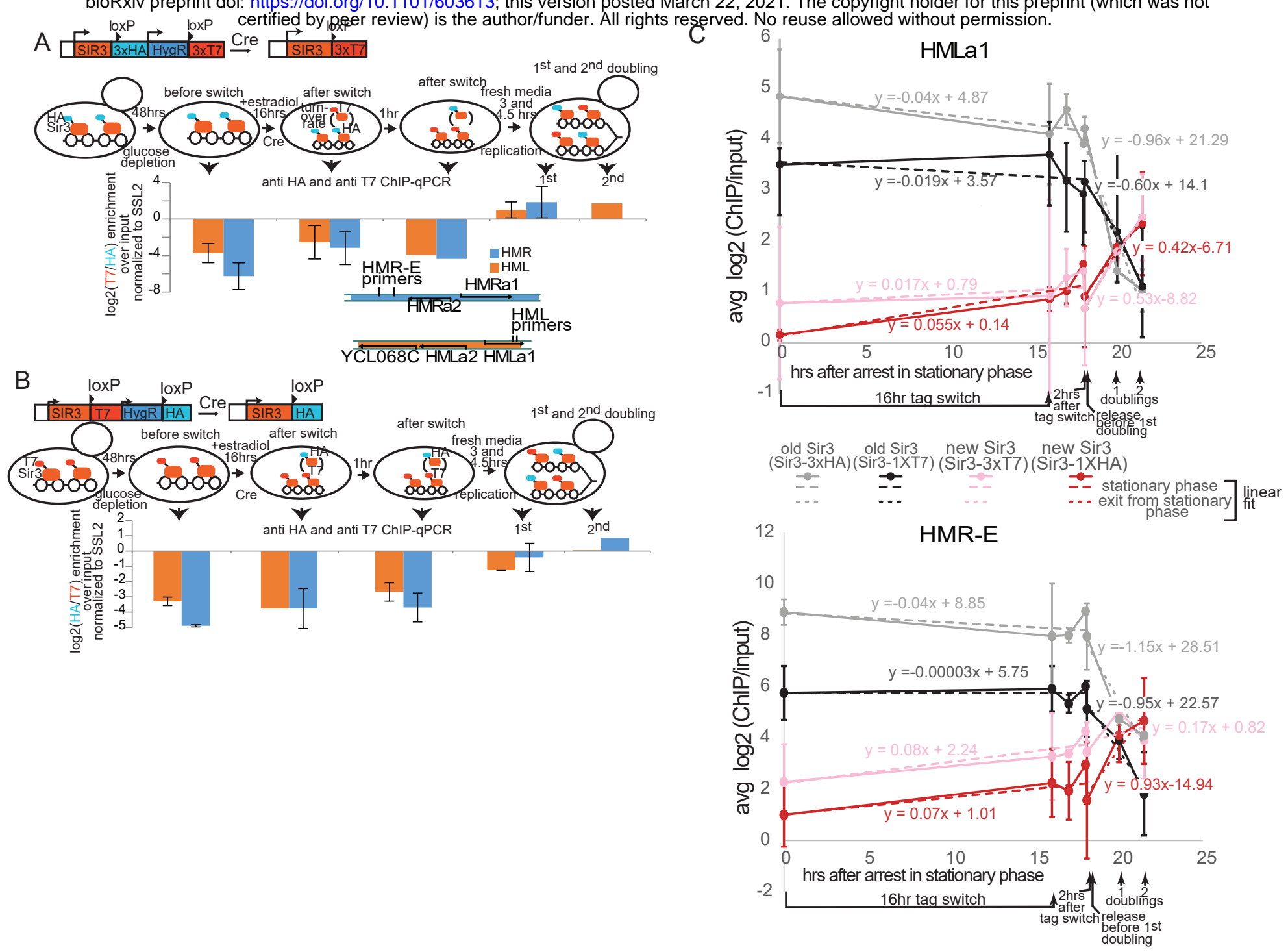

Figure S3: Cell growth dependent and independent Sir3 turnover at silent mating type loci. Sir3 tag switch ChIP qPCR experiments for 3xHA to 3xT7 (A) and 1xT7 to 1xHA tag switches (B). Cells are arrested by glucose depletion before the tag switch. The switch is induced with estradiol addition to arrested cells $(98 \%$ of cells have switched after a $16 \mathrm{hr}$ incubation). Cells are then released from arrest with addition of fresh media and allowed to grow for one or two doublings. Cell aliquots were fixed with $1 \%$ formaldehyde for $20 \mathrm{~min}$ at times indicated in the diagrams and anti-HA and anti-T7 ChIPs were performed on sonicated chromatin. Bar graphs show the ratios of new (T7 in A or HA in B) over old (HA in A or T7 in B) Sir3 enrichment at HMR (blue) and HML(orange) silent mating type loci measured by qPCR (Cts were normalized to the SSL2 locus (where Sir3 does not bind) for ChIPs and inputs, separately, and the ChIP DCts were then normalized to input DCts). The location of qPCR primers are shown in the diagram below the bar graph in A. The bar graphs show the average of two biological replicates (error bars are deviations from the average). C. The scatter plots show the apparent on (change in new Sir3p (ChIP/Input) log2 ratio in $\mathrm{hr}^{-1}$ ) and off (change in old Sir3p (ChIP/Input) log2 ratio in $\mathrm{hr}^{-1}$ ) Sir3p rates at HML (top) and HMR (bottom), calculated from the slopes of linear fits before and after release, as shown. Error bars are standard deviations from the average of two biological replicates. 


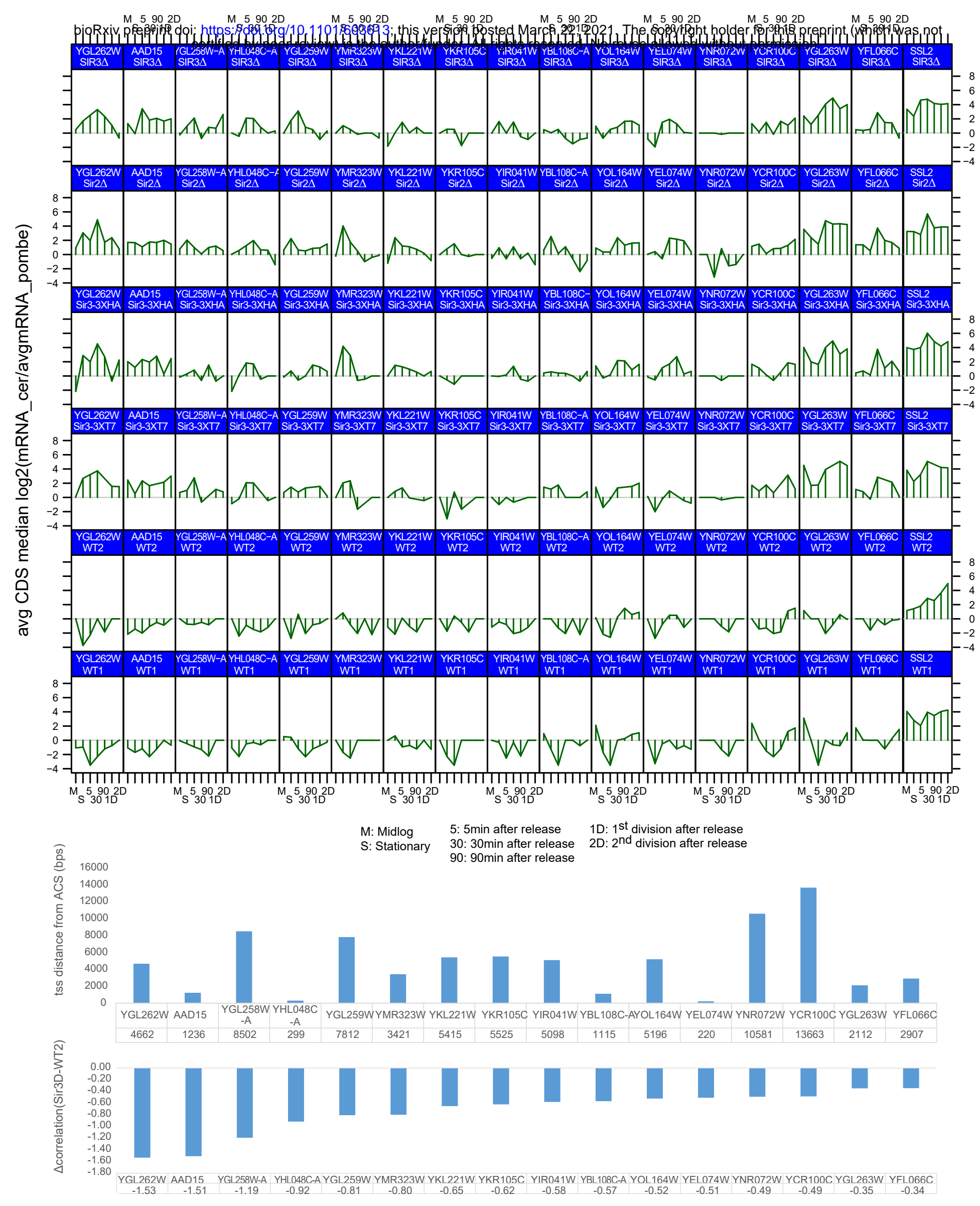

Figure S4: Gene expression dynamics after exit from stationary phase of subtelomeric genes responsive to Sir3 enrichment levels (top). The SSL2 gene is a negative control shown for comparison with Figure 5. We selected genes within 15kbp of subtelomeric Sir3p nucleation sites from the RNA-seq datasets in Figure 6 (middle panel) and kept the genes whose expression profiles were the most different between wt and Sir3D ( $\Delta$ correlation(Sir3 $\Delta$-WT2) between -0.34 and -1.5) as shown in the bar graph on the bottom. 
bioRxiv preprint

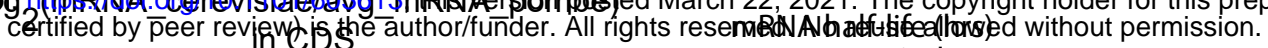

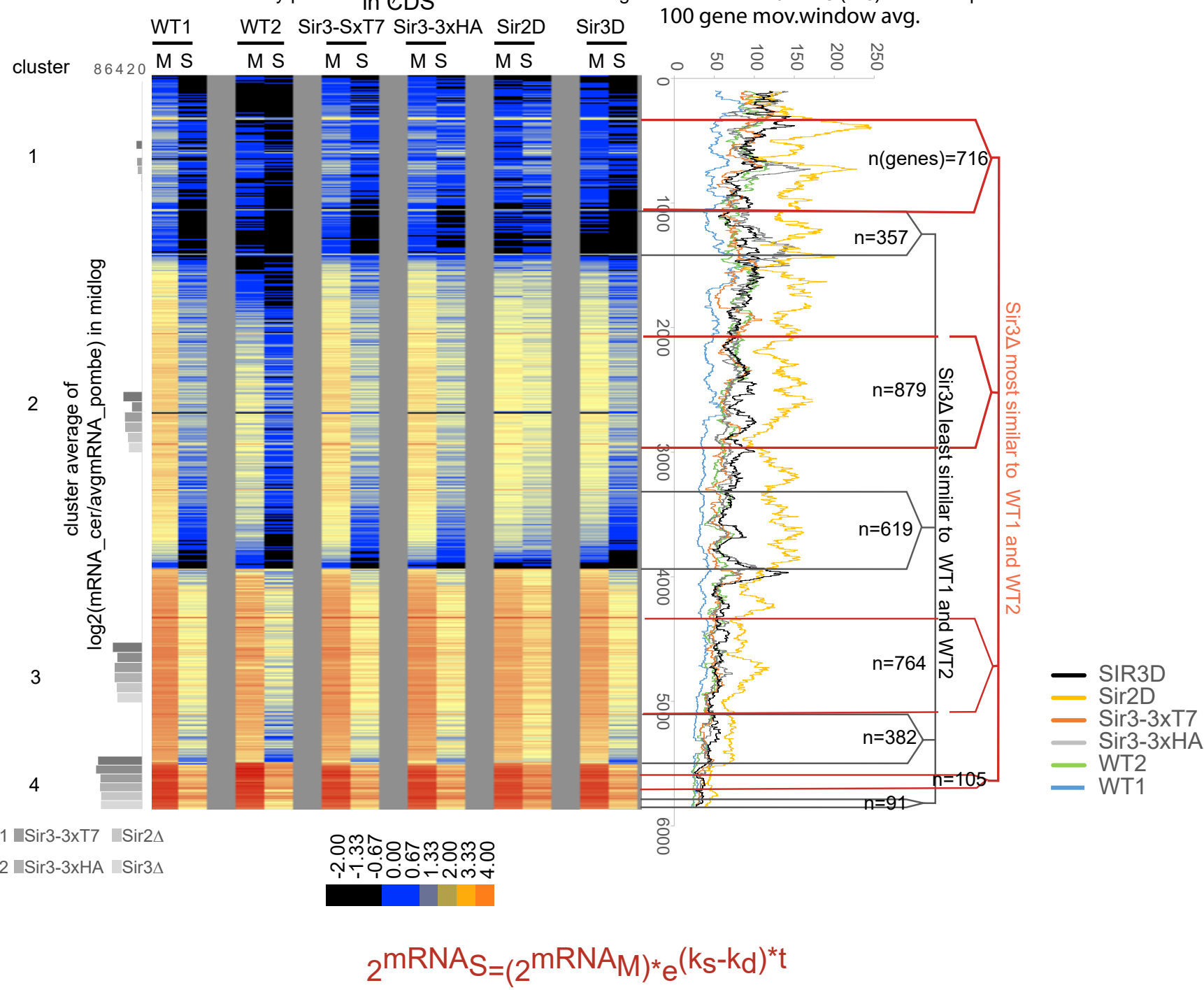

mRNAS=log2(mRNA_cerevisiae/avg_mRNA_pombe) in stationary phase mRNAM=log2(mRNA_cerevisiaelavg_mRNA_pombe) in midlog

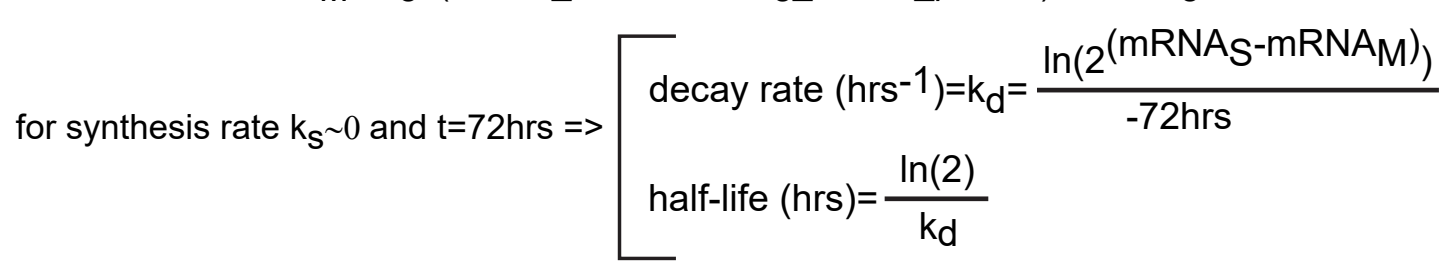

Figure S5: Apparent mRNA half-lives in stationary phase. The heat map shows midlog $(M)$ and stationary (S) phase mRNA levels from the RNA-seq experiment from Figure 6. Genes are organized as in Figure 6A. mRNA decay rates $\left(k_{d}\right)$ and half lives for each gene were determined from the decrease in mRNA levels between midlog $\left(\mathrm{mRNA}_{M}\right)$ and stationary phases (mRNAS) according to the equation shown in red below the heat map. We assumed that mRNA synthesis and decay are exponential and that the mRNA synthesis rate $\left(k_{S}\right)$ is close to 0 in stationary phase for the majority of yeast genes. A hundred gene window moving average of mRNA half-lives for genes and strains in the heat map is shown on the right of the map. The apparent mRNA half lives are globally longer in the Sir2 $\Delta$ strain, but the Sir $3 \Delta$ and Sir3 hypo-morph strains are not significantly different from wt. 